\title{
Model of traveling ionospheric disturbances
}

\author{
Yury P. Fedorenko*, Oleg F. Tyrnov, Vladimir N. Fedorenko, and Vasiliy L. Dorohov
}

Kharkiv V. N. Karazin National University, Kharkiv, Ukraine

*Corresponding author: FedorenkoYP@mail.ru

Received 19 September 2011 / Accepted 5 September 2013

\begin{abstract}
A multiscale semi-empirical model of traveling ionospheric disturbances (TIDs) is developed. The model is based on the following assumptions: (1) TIDs are generated by acoustic-gravity waves (AGWs) and propagate as pressure waves; (2) time intervals between adjacent extrema of atmospheric pressure oscillations in a disturbance source are constant; (3) the pressure extrema propagate from the source up to $\sim 14000 \mathrm{~km}$ at a constant horizontal velocity; (4) the velocity of each extremum is determined only by its number in a TID train. The model was validated using literature data on disturbances generated by about 20 surface and highaltitude nuclear explosions, two volcano explosions, one earthquake and by energetic proton precipitation events in the magnetospheric cusp of the northern hemisphere. Model tests using literature data show that the spatial and temporal TID periods may be predicted with an accuracy of $12 \%$. Adequacy of the model was also confirmed by our observations collected using transionospheric sounding. The following TID parameters: amplitudes, horizontal spatial periods, and a TID front inclination angle in a vertical plane are increasing as the distance between an AGW and the excitation source is increasing. Diurnal and seasonal variability of the TID occurrence, defined as ratio of TID events to the total number of observations for the corresponding period, is not observed. However, the TID occurrence was growing from $\sim 50 \%$ in 1987 to $\sim 98 \%$ in 2010 . The results of other studies asserting that the TID occurrence does not depend on the number of sunspots and magnetic activity are confirmed. The TID occurrence has doubled over the period from 1987 to 2010 indicating increasing solar activity which is not associated with sunspot numbers. The dynamics of spatial horizontal periods was studied in a range of 150-35000 km.
\end{abstract}

Key words. semi-empirical model - multiscale traveling ionospheric disturbances - horizontal velocity of extremum - nuclear explosion - volcano explosive eruption - energetic proton precipitation - magnetospheric cusp - transionospheric sounding disturbance occurrence - sunspot number - magnetic activity

\section{Introduction}

Traveling ionospheric disturbances (TIDs) are known to be generated by acoustic-gravity waves (AGWs) propagating in the neutral atmosphere. The TID-track, i.e., the spatial quasi-periodic modulation of electron concentration $N$ along a surface concentric with the earth's surface, leads to modification of radio wave parameters on propagation paths crossing this track. Therefore, a predictive TID model, updatable by current measurements, may be useful for practical applications (e.g., for reduction of navigation measurement errors and for radio wave propagation forecasts).

AGWs of natural origin play an important role in energy and momentum transfer from high latitudes to mid and low latitudes during precipitation of solar wind protons into the magnetospheric cusp (RTO Technical Report 2009; Karpachev et al. 2010) and from equatorial latitudes to higher latitudes during tropical storms (Vadas \& Crowley 2010). Therefore, a study of AGW-TID phenomena is of great scientific value, allowing deeper understanding of the dominant energy redistribution mechanisms in the atmosphere.

Depending on the wavelength (spatial period) $L$, TIDs are usually separated into two classes: large-scale (LS) and medium-scale traveling ionospheric irregularities (MS TID). The LS TIDs are characterized by wavelengths $L>1000 \mathrm{~km}$, temporal periods $T \approx 0.5-4 \mathrm{~h}$, and horizontal velocities $V \approx 0.4$ $1000 \mathrm{~km} \mathrm{~s}^{-1}$ (Hocke \& Schlegel 1996; RTO Technical Report
2009). The corresponding parameters for MS TIDs are $L \approx 100-600 \mathrm{~km}, T \approx 0.25-1 \mathrm{~h}$, and $V<0.25 \mathrm{~km} \mathrm{~s}^{-1}$. It was predicted theoretically (Hocke \& Schlegel 1996) and proven experimentally (Fedorenko et al. 2008, 2010a, 2010b) that minimal wavelengths $L$ for MS TID do not exceed 100-150 km. Research carried out within the last six decades has revealed that MS TIDs occur much more frequently than LS TIDs. With rare exceptions, LS TIDs are moving from polar regions to the equator, they are observed often, but not always, after auroral disturbances during magnetic storms (Francis 1975; Hocke \& Schlegel 1996). Some scientists assume that the above-mentioned TID classification is defined by the nature of their origin. However, our long-term experimental studies, using satellite beacons, allow us to conclude that both LS and MS traveling ionospheric disturbances are of the same nature and really present different phases of disturbance dynamics. Nevertheless, for convenience we will use the LS and MS terminology in this paper.

Due to their large horizontal scales, LS TIDs can only propagate in the earth-ionosphere waveguide. MS TIDs can move parallel to the earth surface in guided wave-mode (e.g., Gossard \& Hooke 1975) as well as in free wave-mode (e.g., Francis 1974). In the latter case, TID waves are reflected by the Earth's surface. Until now, there exists no generally accepted opinion concerning MS TID propagation and an effective layer thickness where disturbances are observed. For example, according to Iyer (1983) and Senior et al. (2006) the layer thickness varies in the range 100-120 km, but our research has shown that the 
thickness was only about $50 \mathrm{~km}$ in most cases, and values $\sim 100 \mathrm{~km}$ were observed only occasionally (Dorohov et al. 2004; Fedorenko et al. 2008, 2010a, 2010b, 2011).

Now, after 66 years since TIDs discovery (Munro 1948), the theory of interaction between AGWs and TIDs is far from being complete, some predictions and deductions have not been confirmed experimentally and have not been understood completely yet. In particular, the growth of wavelength $L$ in a TID train with increasing distance from an AGW source, predicted by existing theoretical models (Francis 1974; Bristow et al. 1994), has not been observed experimentally. According to the above-mentioned theoretical approaches, the amplitude $A_{d}$ of a TID train as well as $L$ grow when the distance from a disturbance source increases. Here $A_{d}=\Delta N / N$ where $\Delta N$ is the difference between background and perturbed value of $N$. Increase of the amplitude $A_{d}$ during TID propagation was demonstrated in recent experimental research (Tsugawa et al. 2007a), however, the authors could not explain the result. These effects have been clearly discovered and analyzed in our numerous experiments carried out under specific heliogeophysical conditions (see Sect. 5). Due to limited measurement accuracy, there exists a misconception among researchers (see, for example, Evans et al. 1983; Ogawa et al. 1988; Tsugawa et al. 2007a, 2007b; Kotake et al. 2007; Song et al. 2011; Otsuka et al. 2013), concerning diurnal and seasonal occurrence of TIDs (see Sect. 5). According to our observations, there are no minima and maxima in diurnal and seasonal TID occurrence. Further, our observations, carried out during 1987-2010, revealed that TID occurrence does not depend on the phases of the solar activity cycle.

The invariability of the propagation velocity of the wave train extrema was deduced from theoretical treatments and was confirmed experimentally 45 years ago for TIDs generated by nuclear tests and high-power volcanic explosions (Row 1967; Breitling \& Kupferman 1967; Kanellakos 1967; Albee \& Kanellakos 1968; Lomax \& Nielson 1968; Roberts et al. 1982a, 1982b; Igarashi et al. 1994). However, some scientists can hardly accept the fact that this velocity is practically constant up to $14000 \mathrm{~km}$. Therefore, up to now this phenomenon was not explained. At the same time, this effect plays a key role in the unified semi-empirical model of AGW-TID, presented in this article.

During about 20 years after TID discovery in 1946 and the first publication (Munro 1948), which described these experiments, a theoretical AGW-TID model has been developed (Row 1967; Cole \& Greifinger 1969; Liu \& Yeh 1971; Yeh \& Liu 1974; Francis 1974; Gossard \& Hooke 1975). Many authors have created this model, or rather its varieties, and throughout decades geophysicists (e.g., Bristow et al. 1994) repeated its main features.

The model is based on linearized equations of geophysical hydrodynamics for an isothermal non-dissipative atmosphere and a point perturbation source. Winds were taken into account only if TIDs propagate in waveguide mode (WGM) (Gossard \& Hooke 1975). The wind velocity and direction were also taken into consideration in a transformation process from free-wave mode (FWM) to waveguide mode. However, almost all these assumptions are violated in processes of AGW excitation and propagation. For example, in nuclear tests, high-power volcano explosive eruptions and earthquakes the overall dimensions of AGW sources may vary from one to ten kilometers. Energetic proton precipitation into the magnetosphere at $60^{\circ}$ magnetic latitude can form a cusp whose length may vary from $\sim 8000$ to
$10000 \mathrm{~km}$, while a cusp length in a night sector is extended along $L$-shell up to 10-12 time zones (Halperin et al. 1990). Therefore, it is no surprise that a TID front, generated by a high-latitude source, has a length that exceeds 2000 kilometers along a parallel in a zonal direction (Fedorenko et al. 1997; Tsugawa et al. 2007a, 2007b; Kotake et al. 2007; Song et al. 2011; Otsuka et al. 2013). A prior guess, asserting that relative changes of atmospheric density at distances of hundreds and thousands of kilometers from the source of local energy release, is much less than unity is quite courageous. Numerous experiments indicate that such approach is not valid, since the TID amplitude $A_{d}$ may vary from ten to several ten percent at large distances from the source and often reaches 70-80\% (Breitling \& Kupferman 1967; Kanellakos 1967; Albee \& Kanellakos 1968; Lomax \& Nielson 1968; Taran et al. 1979; Afraimovich et al. 2005; Tyrnov et al. 2005; Yakovets et al. 2008; Fedorenko et al. 2010a, 2010b, 2011, 2012). Thus, these approximations may be applicable and reasonable only at the first stage of research.

Surprisingly, the past 60 years of investigations have proven that such a simplified TID model, grounded on simplifying and often not fulfilled assumptions, was generally confirmed by experimental data. Although several of its predictions, for example, spatio-temporal dynamics of the AGW train, have not been confirmed experimentally. But some results, reliably confirmed by experiments, have been simply ignored by investigators, probably due to the surprising nature of these results. In particular, the above-mentioned facts, concerning propagation velocity and amplitudes of the AGW-train, have not been analyzed. TID properties are essentially very similar to properties of solitary waves, generated by non-linear processes (e.g., Lonngren \& Scott 1978; Bullough \& Caudrey 1980). A single maximum of solitary waves moves with constant velocity, while each of the AGW extrema moves with its own constant velocity. It is commonly accepted that both AGW-TIDs and solitary waves propagate over large distances without essential modification of their form and amplitude. A solitary-like TID behavior was once recorded when one TID transited through another, and both remained unchanged (Heisler 1958). Therefore, we suppose that the theory of AGW-TID origin and propagation may be based on the soliton theory. Summing up this short critical review, we may conclude that a complete AGWTID theory does not exist. There exist only two reasonable hypotheses, but their application to particular TID observations sometimes does not give good results.

The nature of regularly observed TIDs, generated by highlatitude natural energy sources, has not been disclosed yet. For years scientists all over the world have been trying to solve this problem. It is worth mentioning the campaigns for simultaneous TID observations at the high, middle, and low latitudes in both hemispheres by all existing diagnostic means (Hocke $\&$ Schlegel 1996). Over the years of observation, there appeared many theories, describing generation mechanisms of regularly observed AGWs. Nonetheless morphological investigations conducted over the last 15 years have facilitated identification of disturbance sources. For example, only the most probable source, namely energetic particle precipitation into the magnetospheric cusp, was mentioned in RTO Technical Report (2009), but the Joule heating by particle precipitation had been mentioned by investigators many times before this report was published. We have also made the same conclusion. It was reported by Karpachev et al. (2010) that the disturbance source, responsible for the LS AGW, recorded by various 
methods during a substorm observed in 1998, appears to be located in the cusp region.

In our model we combine well-known parameters and relations, obtained in the past by various researchers. Our model links different results from theoretical and experimental morphological studies and can predict spatio-temporal dynamics of TID parameters. Certainly, our model is not yet complete. The model is described in Section 2, selection and justification of model parameters are given in Section 3. Sections 4 and 5 are devoted to model verification using experimental data, taken from available literature and our experimental results, obtained by trans-ionospheric sounding by satellite signals.

Thus, the present article aims at the development of a semiempirical model of AGW-TID generation and propagation and its comprehensive experimental verification.

\section{Description of the AGW-TID model}

Our semi-empirical model is based on the following assumptions:

- atmospheric density oscillations are arising under the influence of an external stimulus;

- time intervals between adjacent pressure extrema are constant;

- each pressure extremum propagates from a source as a wave with a horizontal velocity which is constant up to $\sim 14000 \mathrm{~km}$;

- the speed of each extremum is determined by its index in a TID train.

\subsection{The source parameters}

The AGW source is defined as a volume whose characteristic dimension is equal to $\sim 5 \mathrm{~km}$. Atmospheric pressure oscillates in this volume due to local release of energy and momentum. Oscillations start with an intense decrease of the air pressure in the volume. The initial disturbance, generated by energetic proton precipitation in the magnetospheric cusp, is described as an extended source, consisting of the above-mentioned volume sources, aligned along geomagnetic latitudes in the vicinity of $60-75^{\circ}$ at an altitude of $100-200 \mathrm{~km}$. The time interval between an adjacent pressure minimum and maximum, i.e., the temporal half-period, is $\tau_{0}=3.95 \mathrm{~min}$. Each extremum generates an atmospheric pressure wave propagating along the Earth's surface with velocity $V_{i}$. The velocity of each extremum does not vary over distances up to $14000 \mathrm{~km}$. For sphere-shaped sources the wavefront section in a horizontal plane is a circle. If the source is proton precipitation in the magnetospheric cusp, a section is a line or an arc along a magnetic parallel, oriented along the cusp longitudinal axis. In both cases influence of winds, regional irregularities of temperature and pressure were neglected.

The velocities $V_{i}$ and wave amplitudes $A_{i}$, of the first 16 extrema, normalized by peak value, are given in Table 1 . We postulate that $A_{i}$ and $V_{i}$ do not vary during TID propagation.

\subsection{AGW-TID parameters}

All AGW parameters do not depend upon the geomagnetic field, but TID amplitudes depend on the angle between the
Table 1. Amplitudes $A_{i}$ and velocities $V_{i}$ for extremum index $i$.

\begin{tabular}{lccc}
\hline \hline Extremum & $i$ & $A_{i}$ & $V_{i}\left(\mathrm{~km} \mathrm{~s}^{-1}\right)$ \\
\hline Min & 0 & 0.48 & 11.3155 \\
Max & 1 & 0.83 & 3.0000 \\
Min & 2 & 0.97 & 1.1539 \\
Max & 3 & 1 & 0.6820 \\
Min & 4 & 0.95 & 0.4688 \\
Max & 5 & 0.88 & 0.3571 \\
Min & 6 & 0.8 & 0.2809 \\
Max & 7 & 0.7 & 0.2381 \\
Min & 8 & 0.65 & 0.2000 \\
Max & 9 & 0.55 & 0.1754 \\
Min & 10 & 0.49 & 0.1585 \\
Max & 11 & 0.41 & 0.1415 \\
Min & 12 & 0.35 & 0.1245 \\
Max & 13 & 0.29 & 0.1076 \\
Min & 14 & 0.25 & 0.0906 \\
Max & 15 & 0.22 & 0.0736 \\
\hline
\end{tabular}

AGW wave vector and a plane through the magnetic meridian, and upon the magnetic dip angle in the vicinity of the measuring station. Albee \& Kanellakos (1968), Lomax \& Nielson (1968) have ascertained that if an AGW source is located in the southern magnetic hemisphere and TID measurements are performed southward of the AGW source or northward of the magnetic equator, $\mathrm{AGW}$ train maxima (minima) give rise to electron concentration maxima (minima), i.e., $N_{m}$ increases (decreases) relative to the background value. Thus, an altitudinal profile $N(z)$ is shrunk and $z_{m}$ is decreased.

If TID observations are performed to the north of a source, but to the south of the magnetic equator, the AGW train maxima (minima) give rise to electron concentration minima (maxima) and the altitude profile of $N(z)$ stretches out. In all these cases, the total electron content $N_{0}$ is practically unchanged.

Oscillations of ionized atmospheric components, entrained by an AGW in a direction transverse to magnetic field lines, do not lead to the generation of TIDs. The Lorentz force makes charged particles move only along the geomagnetic field line. In the vicinity of the North geographic pole the magnetic field is directed toward the Earth surface, while in the southern hemisphere it is directed upward. According to our theoretical analysis, carried out without involvement of a model of the Earth's electric field, air pressure maxima (minima) in an AGW correspond to maxima (minima) of $N_{m}$ in a TID, but application of this model will, probably, explain all observable effects. At the same time, the whole range of the above-mentioned experimental observations has not been explained yet. The experimental data collected in the same observation cycle in both hemispheres for the same initiating source are in fact only from the four US nuclear explosions. They were produced in 1962 in the Pacific Ocean near the magnetic equator. From these data, the following empirical relationships are deduced.

If a disturbance source is located in the northern hemisphere, the sign of $\Delta N$ (the difference between TID extremum $N_{m}$ and background $N$ values) is defined by the sign of the following empirical relationship (Albee \& Kanellakos 1968):

$$
J_{\Delta N}=\sin (2 \theta) \cos \left(\varphi_{T I D}\right)+0.1 \sin ^{2}(\theta),
$$

where $\theta$ is the magnetic dip angle which is positive in the northern hemisphere and negative in the southern hemisphere, $\varphi_{T I D}$ is the angle between the AGW wave vector and the magnetic north direction at the observation point. Positive values of $J_{\Delta N}$ are proportional to $\Delta N / N_{m}$. For negative $J_{\Delta N}$ 

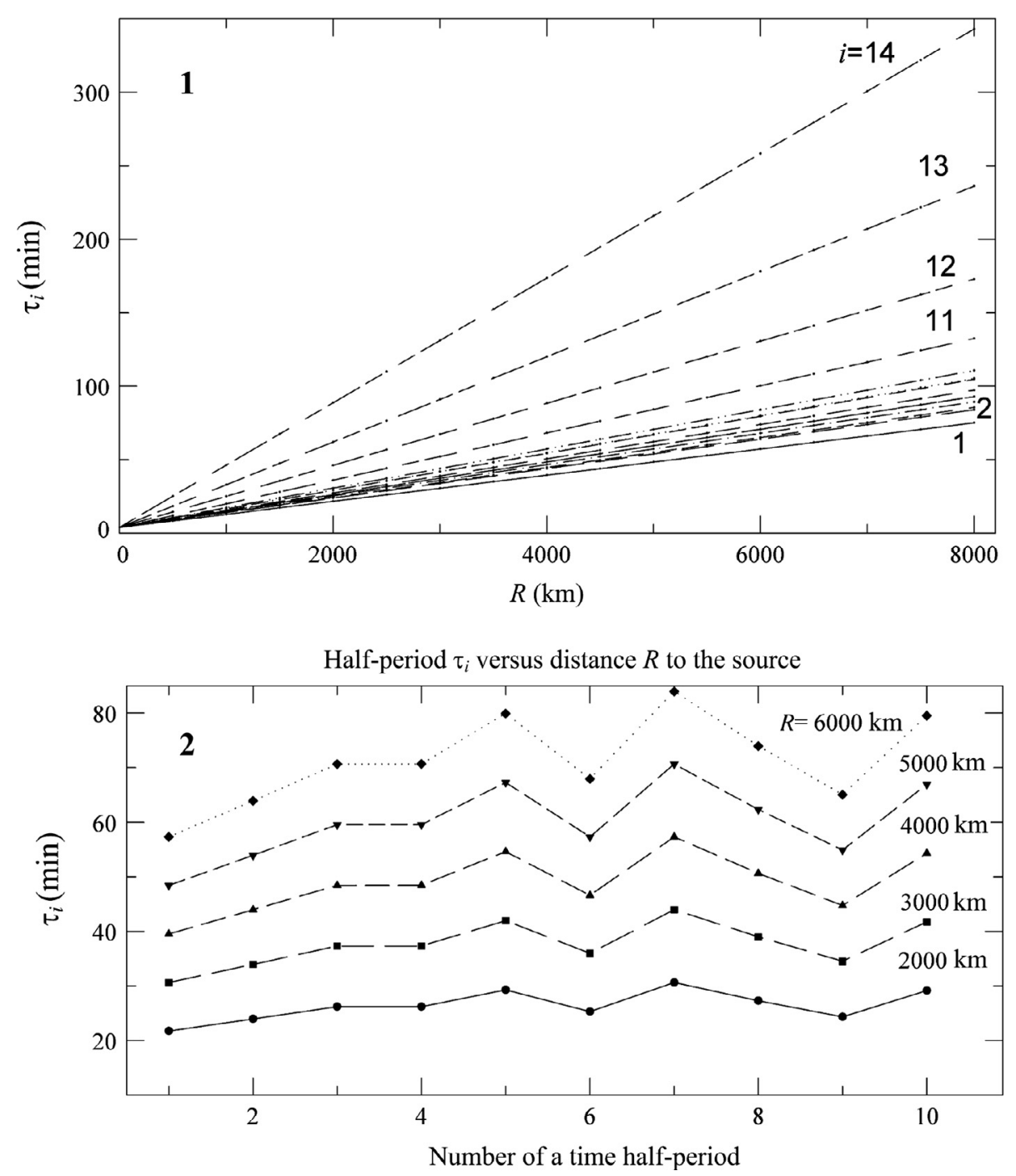

Fig. 1. Temporal half-periods $\tau_{i}$ versus distance $R$ to the source, predicted by the model: panel 1 for $i=1-14$, panel 2 for $i=1-10$.

the value $\left(\Delta N / N_{m}+1\right)$ is proportional to $\exp \left(J_{\Delta N}\right)$. According to our estimates, the formula for $J_{\Delta N}$ was derived using the third, fourth, and fifth TID extrema values given in Table 1. Albee \& Kanellakos (1968) assumed that an AGW is a transverse wave and therefore oscillations in $N$ are accomplished only along a local vertical. Since such extrema generate LS AGWs, we can ascertain that this approximation is valid.

At distance $R$ from a source the time interval $t_{i, i+1}=t_{i+1}-t_{i}$ between adjacent extrema in the AGW train, i.e., the temporal half-period $\tau_{i}$, is calculated using a linear dependence:

$$
\tau_{i}=t_{i, i+1}=\tau_{0}+R\left(1 / V_{i+1}-1 / V_{i}\right)
$$

The sum of $\tau_{i}$ and $\tau_{i+1}$ is the temporal quasi-period $T_{i}$. The curves $\tau_{i}(R)$ for the first 14 quasi-periods are given in Figure 1, panel 1. The reference point for the index $i$ coincides with the first extremum. The curves $\tau_{i}(R)$ for $i$ from 11 to 14 , presenting the longest periods, differ from each other essentially, since they are created by a low-velocity part of an AGW train. The first 10 half-periods $\tau_{i}$ differ from each other less than the other halfperiods. The dependence of $\tau_{i}$ on the extrema index $i$ is not always a monotonic function (see Fig. 1, panel 2).
Excitation of an AGW train starts by generating a minimum whose amplitude is $A_{0}=0.48 A_{3}$ and velocity exceeds $11 \mathrm{~km} \mathrm{~s}^{-1}$. Since the first extremum has a small amplitude and large propagation velocity, it may be difficult to detect it experimentally. An initial disturbance onset results in an air pressure decrease, which, in some cases, especially during a solar activity maximum, may be masked by subsequent extrema having larger amplitudes (up to $\sim 2$ times). Therefore, in our model the zero-time reference is related to the first extremum (maximum) whose amplitude $A_{1}=0.83 A_{3}$ and velocity $V_{1}=3 \mathrm{~km} \mathrm{~s}^{-1}$, although the disturbance onset occurs $3.95 \mathrm{~min}$ before this event. When the second extremum (minimum) is initiated, the first AGW spatial half-period moves away from the source area. When the third extremum (second maximum) is originated, the wave train represents the full quasi-period of the horizontal wave. After origination of the fourth and fifth extrema the wave train contains the two full spatial quasi-periods. Formation of each two subsequent extrema increases the number of quasi-periods in a wave train by unity.

Let a horizontal distance between adjacent AGW train extrema, i.e., a horizontal spatial half-period, be $l_{i}$. Then the sum of adjacent half-periods $l_{i}$ and $l_{i+1}$ is a spatial quasi-period $L_{i}$. According to our assumptions, $l_{i}$ is an increasing function of time: 


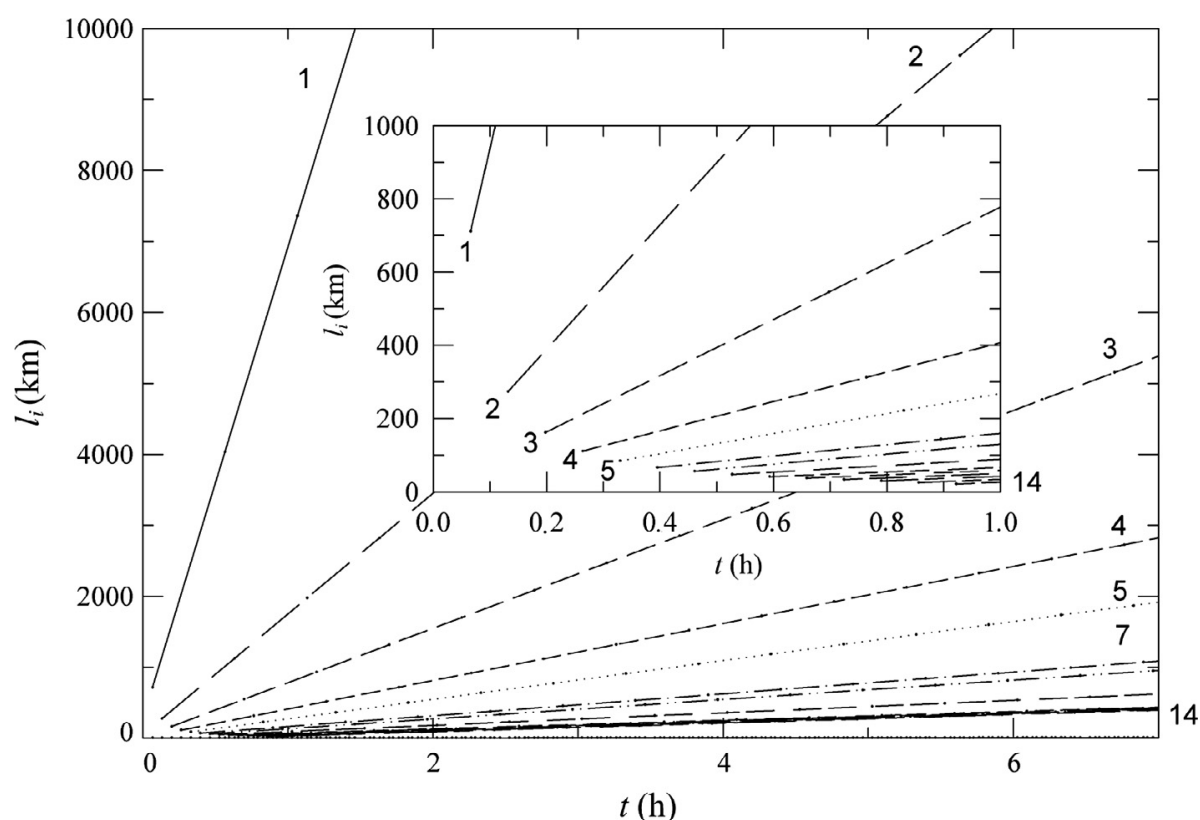

Fig. 2. Spatial half-periods $l_{i}$ versus time $t$, predicted by the model. Zero-time reference corresponds to the time of disturbance initiation.

Table 2. Minimal values of $l_{i}$ and $L_{i}$.

\begin{tabular}{ccccc}
\hline \hline $\begin{array}{c}\text { Index of } l_{i} \text { in } \\
\text { AWG train }\end{array}$ & $\begin{array}{c}\text { Origination } \\
\text { time }(\mathrm{min})\end{array}$ & $\begin{array}{c}l_{i} \\
(\mathrm{~km})\end{array}$ & $\begin{array}{c}\text { Index of } L_{i} \text { in } \\
\text { AWG train }\end{array}$ & $\begin{array}{c}L_{i} \\
(\mathrm{~km})\end{array}$ \\
\hline 1 & 3.95 & 711 & & \\
2 & 7.9 & 273.5 & 1 & 984.5 \\
3 & 11.85 & 161.6 & & \\
4 & 15.8 & 111.1 & 2 & 272.7 \\
5 & 19.75 & 84.6 & & \\
6 & 23.7 & 66.6 & 3 & 151.2 \\
7 & 27.65 & 56.4 & & \\
8 & 31.6 & 47.4 & 4 & 103.8 \\
9 & 35.55 & 41.6 & & \\
10 & 39.5 & 37.6 & 5 & 79.1 \\
11 & 43.45 & 33.5 & & \\
12 & 47.4 & 29.5 & 6 & 63.1 \\
13 & 51.35 & 25.5 & & \\
14 & 55.3 & 21.5 & 7 & 47 \\
\hline
\end{tabular}

$$
l_{i}=V_{i} \tau_{0}+\left(V_{i}-V_{i+1}\right) t^{\prime},
$$

where $t^{\prime}=t-i \tau_{0}$. The half-period $l_{i}$ arises at time $t^{\prime}=0$. Figure 2 shows $l_{i}(t)$ for the first 14 quasi-periods, calculated using equation (3) and data presented in Table 1 . The minimum lengths of $l_{i}$ and $L_{i}$ are observed at the moment of their initiation. The data for the first fourteen half-periods and seven quasi-periods are given in Table 2. For example, the fifth half-period, whose length is $79.1 \mathrm{~km}$, originates at the 40th minute after disturbance offset. In time, the lengths of half-period $l_{i}$ and quasi-period $L_{i}$ increase linearly from the lowest values observed at origination.

Further, we assume a TID train consisting of the first fourteen half-periods. When an AGW train moves away from a disturbance source, its half-periods $l_{i}$ and quasi-periods $L_{i}$ are linearly increasing. Let $R_{1}$ be a distance between a disturbance source and the first extremum in a TID train. The $l_{i}$ and $L_{i}$ for the first extrema at distances $R_{1}=1500$ and $3000 \mathrm{~km}$ are given in Table 3 and Table 4. These values of $R_{1}$ are observed 8.3 and $16.7 \mathrm{~min}$ after generation of the first extremum. The selected values of $R_{1}$ are typical for the observations (see Sects. 4 and 5).
Table 3. $l_{i}$ and $L_{i}$ for $R_{1}=1500 \mathrm{~km}$.

\begin{tabular}{crcc}
\hline \hline Index $i$ & $l_{i}(\mathrm{~km})$ & Index $i$ & $L_{i}(\mathrm{~km})$ \\
\hline 1 & 1196.5 & & \\
2 & 285.7 & 1 & 1482.3 \\
\hline
\end{tabular}

Table 4. $l_{i}$ and $L_{i}$ for $R_{1}=3000 \mathrm{~km}$.

\begin{tabular}{lrcc}
\hline \hline Index $i$ & $l_{i}(\mathrm{~km})$ & Index $i$ & $L_{i}(\mathrm{~km})$ \\
\hline 1 & 2119.6 & & \\
2 & 521.7 & 1 & 2641.3 \\
3 & 223.3 & & \\
4 & 116.9 & 2 & 340.2 \\
\hline
\end{tabular}

Apparently, at distances $1500-3000 \mathrm{~km}$ the first period of the wave train presents an LS TID and the next periods are MS TIDs. The smallest value of $L_{i}$ for the first extremum at such distances is more than $300 \mathrm{~km}$.

\subsection{The layer height and thickness}

LS AWG-TIDs are observed in the Earth-ionosphere waveguide, whose effective thickness is $2 H=160 \mathrm{~km}$ and a maximum TID amplitude is observed at the height $z_{m}$ of the F2 layer maximum. MS AGWs propagate in free-wave mode and after reflection from the Earth's surface at different angles and generate at ionospheric heights MS TIDs which are observed in a layer with effective thickness $2 H=50 \mathrm{~km}$. The incidence and reflection angles are the greater the farther the reflection point from the disturbance source is.

\subsection{Spatial scans of half-periods}

For a given value of $R_{1}$, the distance $R_{i}$ between the disturbance source and the nearest end of half-period $l_{i}$ in an AGW train is given by the expression: 

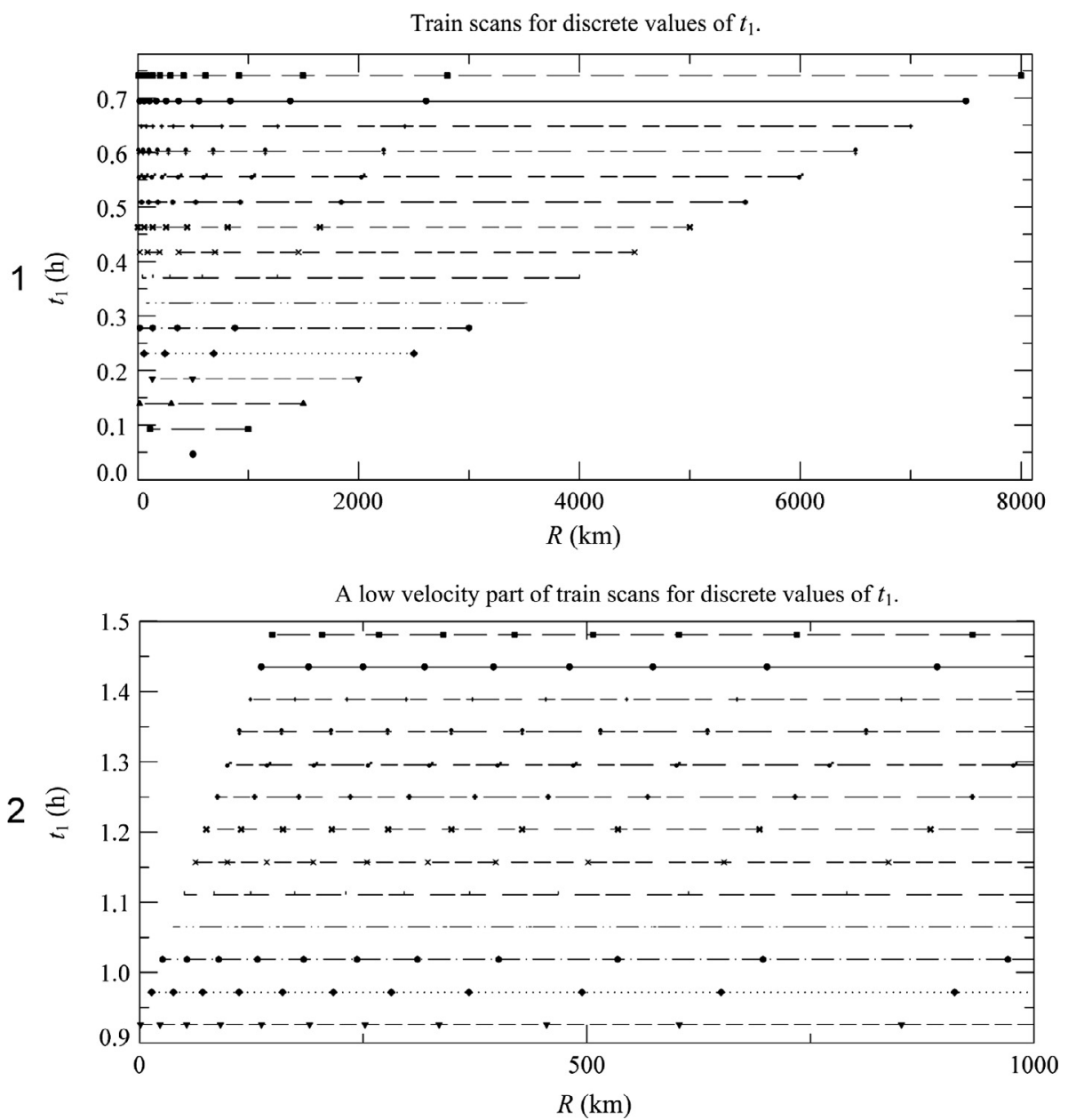

Fig. 3. Space-time dynamics of AGW train, consisting of 14 half-periods.

$$
\begin{gathered}
R_{i}=R_{1}-\sum_{i=1}^{i} l_{i}, \\
l_{i}=V_{i} \tau_{0}+\left(V_{i}-V_{i+1}\right) t_{i}^{\prime}, \\
t^{\prime}{ }_{i}=t_{1}-i \tau_{0} .
\end{gathered}
$$

The spatio-temporal dynamics of half-periods $l_{i}$ for different times $t_{1}$ are shown in Figure 3, where positions of half-periods $R_{i}$ and the first extremum $R_{1}$ are marked by markers on rectilinear segments, parallel to the axis $R$. This plot is known as the spatial scan of half-periods $l_{i}(R)$.

Panel 1 of this figure clearly shows the strong difference between the lengths of the first four half-periods of the TID train, which are generated by the first five high-speed extrema (recall that $V_{0}$ is not included in the calculations). The half-periods $l_{i}$, generated by low-speed extrema of the TID train, are shown in Figure 3, panel 2. All fourteen half-periods are formed at time $t_{1}=0.93 \mathrm{~h}$, and only for $t_{1} \geq 0.97 \mathrm{~h}$ the left boundary of the fourteenth half-period and the whole TID train starts to move away from the excitation source. Thus, the differences between adjacent $l_{i}$ values in the low-speed TID train part are much smaller than those in the high-speed part.

Figure 4 shows the plots $L_{i}(R)$ for distances $R_{1}=500$ $8000 \mathrm{~km}$. Here $R$ is the distance from the source to the middle of each quasi-periods. As can be seen $L_{i}(R)$ is an almost linear function in the range $R=1000-1500 \mathrm{~km}$ for the train part nearest to the disturbance source (corresponding to MS AGWs).
To analyze space-time dynamics of AGW quasi-periods we performed a thought experiment. Let there exist a hypothetical observer capable of measuring visible AGW quasi-periods at predefined moments. The observer sequentially settles at measuring stations located at distances $R_{S}$ from the source. As soon as a left boundary of a nearest to the source quasi-period crosses the vertical line at the observer's site, the observer starts measuring the next quasi-period, which is excited nearer to the source than the previous quasi-period. To allow the observer to perform the measurements, we calculated the $L_{i}(R)$ scans, i.e., a set of quasi-periods for the set of $t_{1}$.

Figure 5 shows the graphs $L(t)$ plotted with increments $\Delta R_{S}=500 \mathrm{~km}$ for the range $R_{S}=500-3500 \mathrm{~km}$ where according to estimates the cusp can be located in our measurements (see Sect. 5). At first glance, saw-tooth pieces on the curve $L(t)$ most notably observed until a minimum value of $L$ followed by almost monotonic increase indicate model inadequacy. In fact, such behavior should be observed at nearly continuous measurements of AGW spatial periods over a measuring station for different distances from a disturbance source.

To explain the results obtained by the observer at various distances $R_{S}$ we will analyze the $L(t)$ dynamics at $R_{S}=500 \mathrm{~km}$ (Fig. 5, panel 1). The first value of $L=1482 \mathrm{~km}$ corresponds to a time delay $t=0.138 \mathrm{~h}(8.3 \mathrm{~min})$ relative to the time of the first extremum excitation. At that moment the first extremum is at distance of $1500 \mathrm{~km}$ from a source, and the first quasi-period consists of the first and second half-periods. According to the measurements of our hypothetical observer the first 
Y.P. Fedorenko et al.: Model Traveling Ionospheric Disturbances
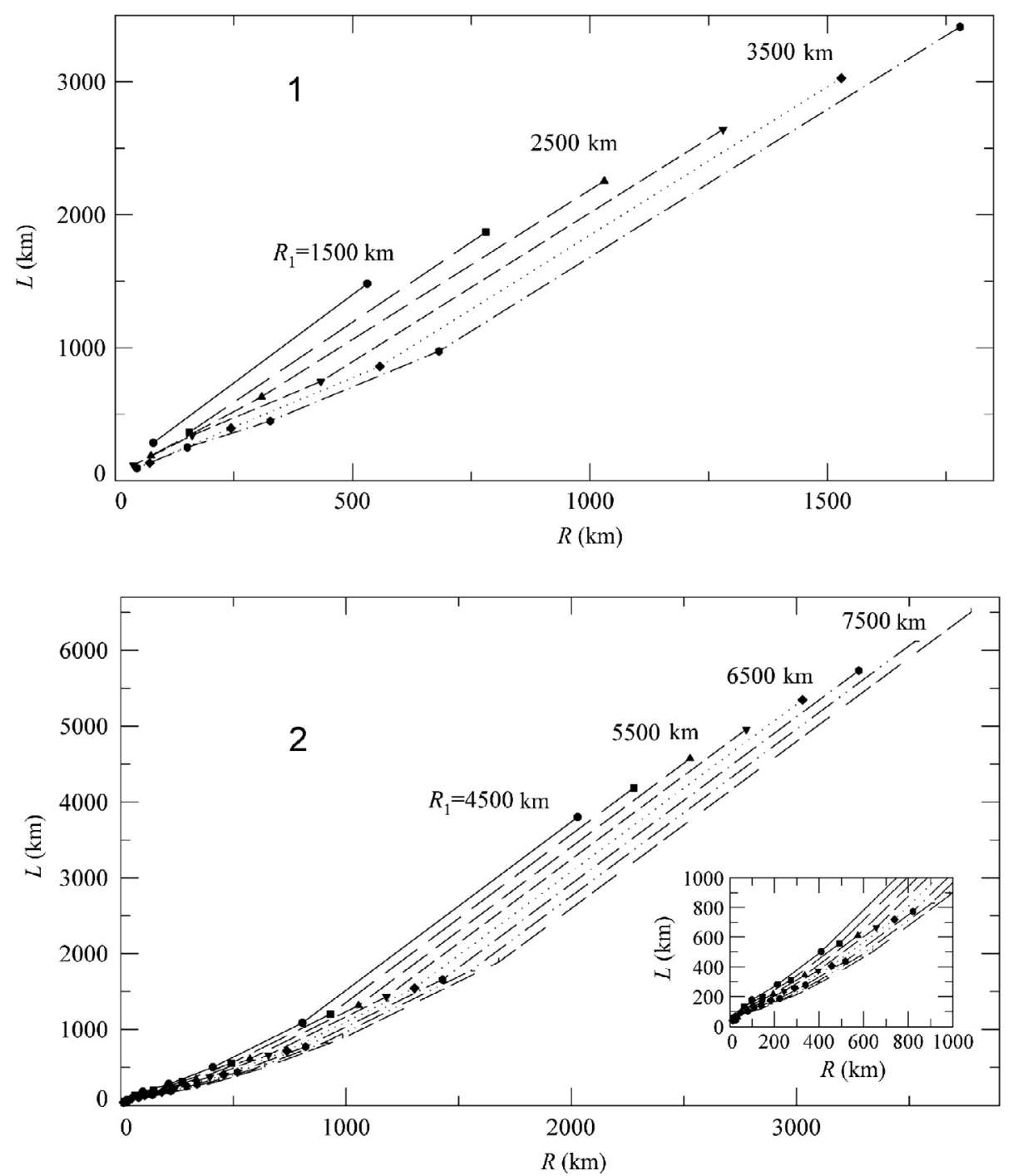

Fig. 4. Spatial half-periods $L$ versus distances to a disturbance source $R$ for various values of $R_{1}$.

quasi-period as well as the next quasi-periods increases linearly as they propagate over the measuring station. This phase of the AGW movement over the measuring station corresponds to the first, linearly increasing part of the plot (up to $\sim 3027 \mathrm{~km}$ at $t=0.324 \mathrm{~h}$ ). As soon as the initial part of the second quasiperiod, i.e., the right boundary, crosses the observer's vertical, the measured period will consist of the second and third halfperiods, either of the two being much shorter than the first quasi-period. As a result this period, consisting of the second and third half-periods, is considerably shorter. In our example its length is about $973 \mathrm{~km}$. This new period is also linearly increasing up to $\sim 1200 \mathrm{~km}$, and it changes in a stepwise manner to a considerably smaller quasi-period which consists of the third and the fourth half-periods and is about $611 \mathrm{~km}$ long.

Thus, while the AGW is moving over the measuring station, the observed quasi-period and its temporal gradient in each saw-toothed fragment of the plot $L(t)$ are becoming smaller and smaller, since differences between adjacent values of $V_{i}$ in the train are reduced. This process continues until a minimum quasi-period $L_{\text {min }}=200 \mathrm{~km}$ is reached. Then almost monotonic increase $L(t)$ is observed, since the differences between the adjacent extrema with indexes $i>8$ are practically constant.
The experimental observation of the saw-tooth effect is illustrated in Section 5.

\subsection{Slope of the AGW front in a vertical plane}

The inclination of the TID front, defined by an angle $\xi$, measured in a vertical plane, is determined by:

$$
\begin{gathered}
\xi=\tan ^{-1}\left(R /\left(z_{m}+z_{0}\left(R_{E}+z_{m}\right) /\left(R_{E}+z_{0}\right)\right)\right), \\
\xi=2.1 \times 10^{-4} R,
\end{gathered}
$$

where $R_{E}$ is the Earth's radius, $z_{0}$ is the source altitude over the Earth surface, and the distance $R$ is determined at the F2-layer maximum height. The plots $\xi(R)$ for both propagation modes are shown in Figure 6. Calculations of $\xi(R)$ for the free-wave mode were made using following parameters: the TID height is $z_{m}=300 \mathrm{~km}$ and disturbance source heights are $z_{0}=100$ and $200 \mathrm{~km}$ (curves 1 and 2, respectively). The distance $R$ refers to a height $z_{m}=300 \mathrm{~km}$. 
J. Space Weather Space Clim. 3 (2013) A30
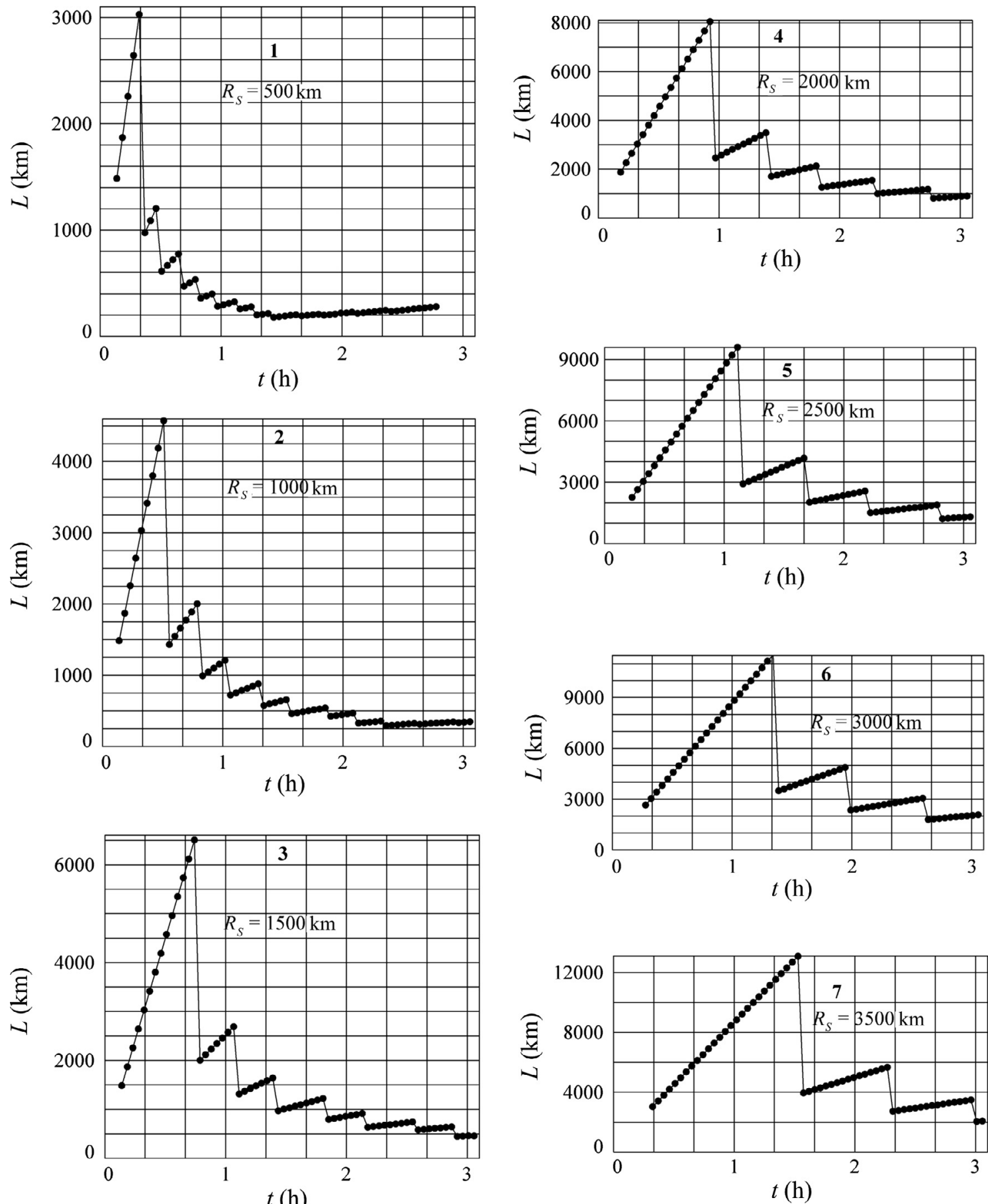

$t(\mathrm{~h})$

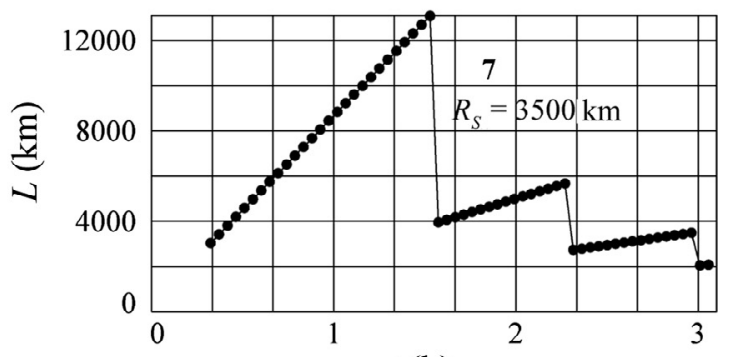

$t(\mathrm{~h})$

Fig. 5. Quasi-period $L$ versus time $t$ for various distances $R_{S}$ between the measuring station and TID sources. Zero-time reference corresponds to disturbance initiation time.

\subsection{Spatial distribution of electron density}

If a TID propagates in a direction defined by an azimuthal angle $\alpha$, a spatial modulation of electron density $N$ in a horizontal plane is described by:

$$
\begin{gathered}
N=N_{n}\left[1+A_{d} F(z) F_{1}(\varphi, \lambda)\right], \\
F(z)=\exp \left[-\frac{\left(z-z_{m}\right)^{2}}{H^{2}}\right],
\end{gathered}
$$

$$
\begin{gathered}
F_{1}(\varphi, \lambda)=\sin \left[m_{\varphi}(\varphi-\Delta \varphi)+m_{\lambda}(\lambda-\Delta \lambda)+\Phi\right] \\
\Delta \varphi= \pm \frac{z-z_{m}}{R_{E}+z_{m}} \tan \xi, \Delta \lambda= \pm \frac{z-z_{m}}{R_{E}+z_{m}} \tan \xi \\
m_{\varphi}=\frac{2 \pi}{L}\left(R_{E}+z_{m}\right) \cos \alpha, m_{\lambda}=\frac{2 \pi}{L}\left(R_{E}+z_{m}\right) \sin \alpha,
\end{gathered}
$$


Y.P. Fedorenko et al.: Model Traveling Ionospheric Disturbances

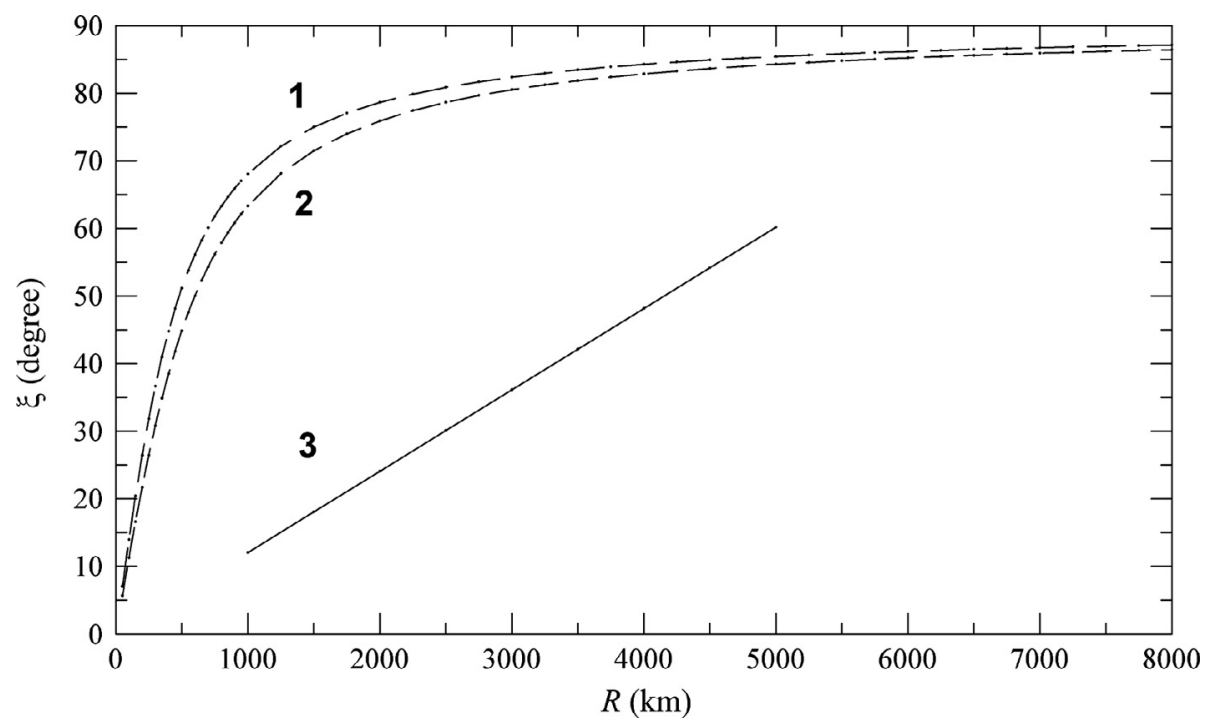

Fig. 6. Inclination angles $\xi$ versus $R: 1-$ MS TID, $z_{0}=100 \mathrm{~km} ; 2-$ MS TID, $z_{0}=200 \mathrm{~km} ; 3-$ LS TID.

$$
\xi=\xi_{50}-\left(\varphi-\varphi_{0}\right) k_{\xi}, \varphi_{0}=50^{\circ} \mathrm{N},
$$

where $\varphi, \lambda$ are geographic latitude and longitude of the measuring station, respectively; $\xi_{50}$ is the inclination angle at $\varphi=\varphi_{0} ; A_{d}=A_{d 50}-\left(\varphi-\varphi_{0}\right) k_{A d}, N_{n}$ is the undisturbed spatial distribution of electron density at a given moment, defined by a global ionospheric model, $A_{d 50}$ is the relative amplitude of a TID at $\varphi=\varphi_{0}, \Phi$ is the initial phase shift of the corresponding disturbance harmonic, $k_{A d}$ and $k_{\xi}$ are proportionality coefficients, and $z$ is the height over the Earth's surface. Plus or minus signs in the formulas for $\Delta \varphi$ and $\Delta \lambda$ are selected depending upon the relative position of the TID source and $\mathrm{m}$. If the source is to the north or west of the measuring station, minus signs are used. Otherwise, plus signs are used. The functions $F(z)$ and $F_{1}(\varphi, \lambda)$ describe modifications of TID amplitude and phase depending on $z, \varphi$ and $\lambda$. The global ionospheric model may be improved using navigation satellite signals (Tyrnov et al. 2005).

\section{The choice of the model parameters}

The model is based on the following postulates:

- extrema periodicity;

- space-time constancy of $V_{i}$ and $A_{i}$;

- invariability of $\tau_{0}$ during excitation of 16 train extrema.

The key model parameters are:

- extrema velocities $V_{i}$;

- relative amplitudes of train extrema $A_{i}$;

- half-period of atmospheric pressure oscillations $\tau_{0}$ in an excitation source;

Due to the high velocities of the first extrema (see Table 1) accurate experimental values can be obtained only by continuous monitoring of ionospheric parameters, in particular, the total electron content or $N_{m}$. Such approach was implemented by Roberts et al. (1982a, 1982b). In the first article the total electron content was monitored by measuring the Faraday rotation angle of linearly polarized VHF signals radiated by geostationary satellites. The experiment detected a disturbance initiated by a volcanic explosion (eruption of the volcano St. Helens, on May 16, 1980). These data were used to adapt the model to an isothermal atmosphere disturbance by a point source (Cole \& Greifinger 1969; Liu \& Yeh 1971). As a result, parameters of a simplified global equivalent isothermal atmosphere have been determined, namely: the speed of sound $C=486 \mathrm{~m} / \mathrm{s}$, adiabatic index $\gamma=1.38$; the free-fall acceleration due to gravity averaged over height range from 0 to $350 \mathrm{~km} \mathrm{~g}=9.33 \mathrm{~m} / \mathrm{s}^{2}$.

The aforementioned model, equivalent isothermal atmosphere adapted, as it is named by Roberts et al. (1982a) was successfully tested by Roberts et al. (1982b), using experimental data obtained after the explosion of the volcano St. Helens. For the diagnosis of disturbances the Faraday effect of pulsar PSR $0329+54$ signals, recorded by a highly sensitive radio interferometer, was used.

Using data presented in the latter article, we calculated $V_{i}$ values for $i$ from 1 to 11 . The $V_{i}$ values for $i$ from 12 to 15 are derived as linear extrapolation of $V_{i}$ values for $i$ from 9 to 11. $V_{0}$ was found via an extrapolation of ninth-order regression for $V_{i}$ values for $i$ from 1 to 11 . In what follows all $V_{i}$ values for $i$ from 0 to 15 will be called the $V_{i}$ model (the label Model in Figure 7). The data thus obtained are given in Table 1. The space-time constancy of $V_{i}$ values was experimentally confirmed by Breitling \& Kupferman (1967), Row (1967), Kanellakos (1967), Albee \& Kanellakos (1968), Lomax \& Nielson (1968), Roberts et al. (1982a, 1982b), Igarashi et al. (1994) for distances from the disturbance source up to $\sim 14000 \mathrm{~km} . A_{i}$ values are taken from Bristow et al. (1994), calculated for a delay relative to the onset of AGW excitation equal to $3.333 \mathrm{~h}$. This delay is close to the average value used in our experimental data.

We postulated invariability of $\tau_{0}$ during generation of all 16 AGW extrema. Following Albee \& Kanellakos (1968), we have used the formula $\tau_{0}=2 \pi C /(\gamma g)$ (Row 1967). We substitute the parameters from an equivalent isothermal atmosphere adopted model (Roberts et al. 1982a) and obtained $\tau_{0}=3.95 \mathrm{~min}$. 
J. Space Weather Space Clim. 3 (2013) A30

(a)
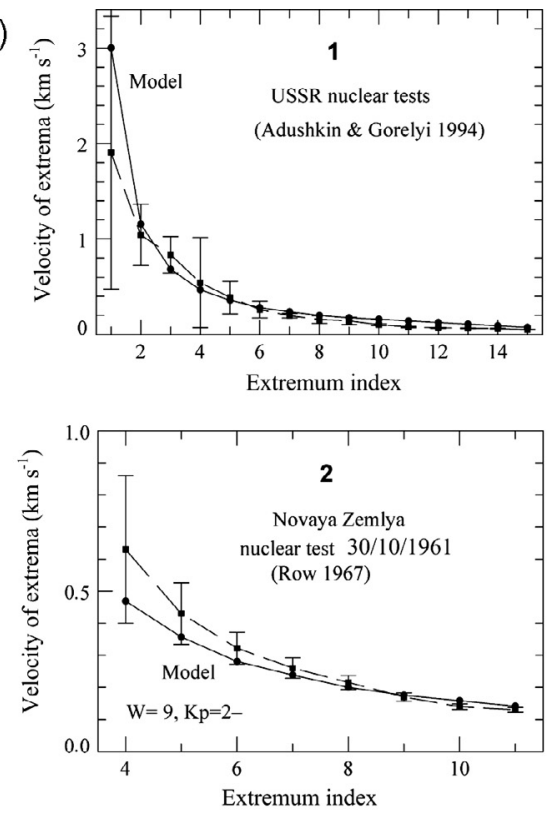
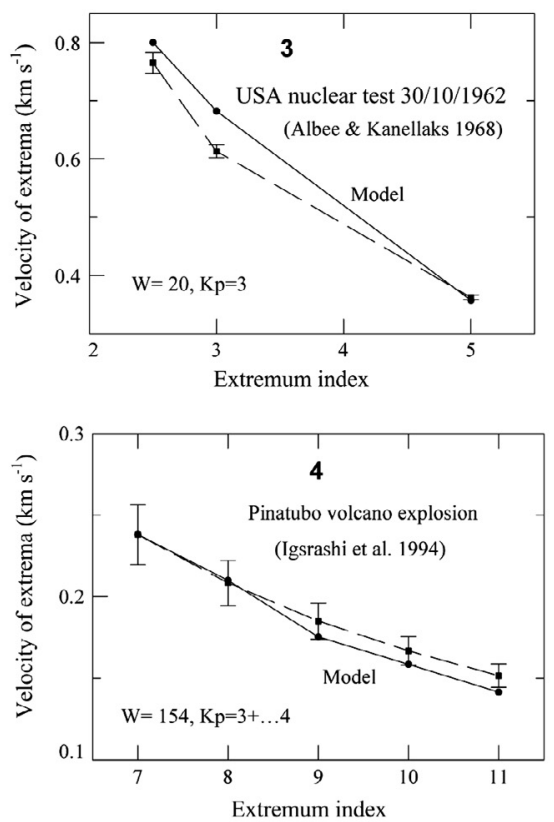

Extremum index

Extremum index: odd numbers correspond to maximum, even number correspond to minimum

(b)

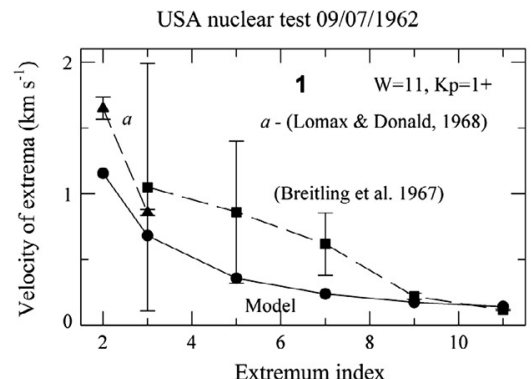

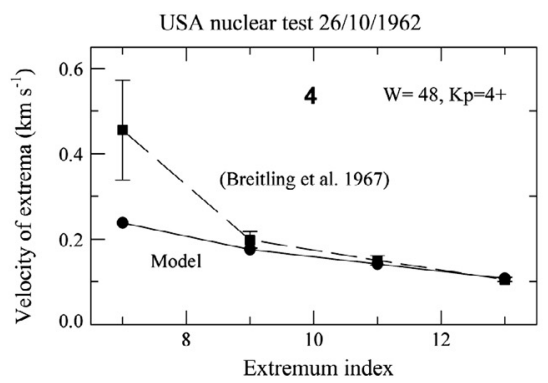
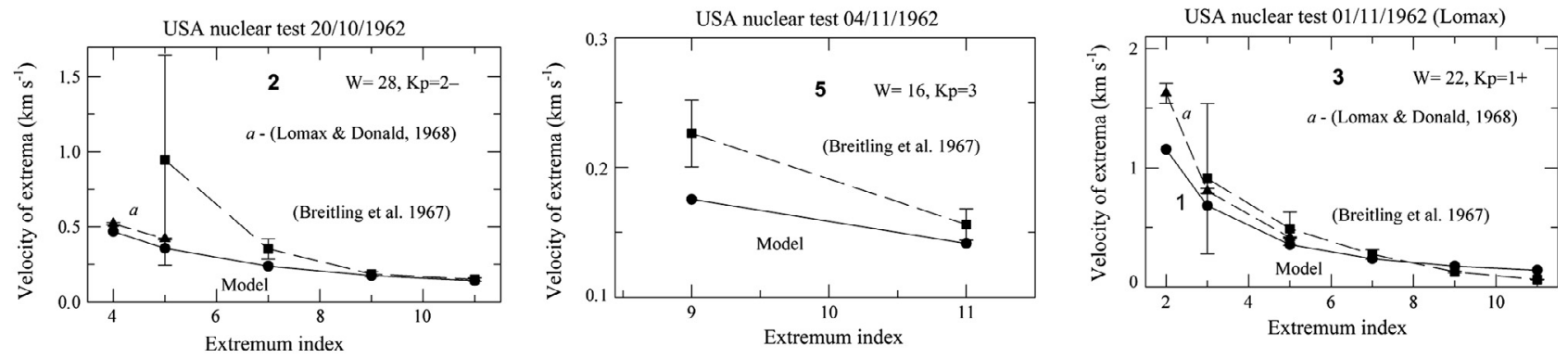

Extremum index: odd numbers correspond to maximum, even number correspond to minimum

Fig. 7. (a) Extrema velocities of a TID train versus its index for surface nuclear explosions (panels 1, 2, and 3) and volcano eruption (panel 4). (b) Extrema velocities of TID train versus its index for high-altitude nuclear explosions.

The slope of the AGW-TID front in the vertical plane for the MS TID (5) and LS TID (6) was obtained from Fedorenko et al. (2010b). The relation (5) was obtained for propagation of omnidirectional waves in a spherically symmetric medium without refraction. The relation (6), valid for the waveguide mode of AGW propagation, was obtained from Kunitsyn et al. (2007).

The values of $2 H$ for the MS and LS TID, the height of the waveguide longitudinal axis for the LS TID, and the layer in which the MS TID are observed, were inferred from transionospheric sounding using signals of Russian low-orbital navigation satellites (Cicada and Cicada-M), and from incoherent scatter radar data (Fedorenko et al. 2008, 2010a, 2010b, 2011, 2012).

\section{Model validation using literature data}

\subsection{Velocities of extrema}

An estimate of the onset velocity $V_{0} \approx 10 \mathrm{~km} \mathrm{~s}^{-1}$ (in the model $V_{0} \approx 11.3 \mathrm{~km} \mathrm{~s}^{-1}$ ) is given only in one single publication (Taran et al. 1985). The $V_{0}$ value was estimated from incoherent scatter radar observations at a distance of $3500 \mathrm{~km}$ from the source of a surface explosion of yield $Q \approx 0.24$ Mt TNT, 
detonated in the USSR on 28/11/1981 at 02:31 UT. Since the onset speed is difficult to measure experimentally, we could observe only the consequences of the initial disturbance passage. Therefore we could determine the extremum velocities $V_{i}$, starting from $V_{1}$.

In Figure 7a, panel 1 , the values $V_{i}$ for $i$ from 1 to 15 given in Table 1 (solid line) are compared with our calculations based on the experimental data presented by Adushkin \& Gorelyi (1994) (dashed line). In this paper the averaged data, obtained after 27 nuclear explosions with equivalent yields $Q$ from 5 to 10 and from 20 to $30 \mathrm{Mt} \mathrm{TNT}$, are given. In the figure, only data for the second range of $Q$ are presented. All the explosions were made at Novaya Zemlya during mass testing of nuclear weapons in the USSR. Explosion heights were unknown. However, we suppose the heights did not exceed $\sim 5 \mathrm{~km}$. Averaging was carried out by the method of epoch superposition. The critical frequencies of the F2-layer $\left(f_{0} \mathrm{~F} 2\right)$ were recorded using ionosondes at two measuring stations $\left(R_{S}=1000\right.$ and $\left.R_{S}=4000 \mathrm{~km}\right)$ in increments of $15 \mathrm{~min}$, starting from the moment of the explosion. The arrival times of the first three extrema for $R_{S}=1000 \mathrm{~km}$ in accordance with our $V_{i}$ model should be 5.6, 14.4, and $24.4 \mathrm{~min}$. Therefore, we believe that these extrema could not be detected by this station. The $V_{i}$ values for extrema from 1 to 3 were determined using data obtained by the second station, and for extrema from 10 to 15 using data collected by the first station. The values for extrema from 4 to 9 were obtained by averaging data from both stations.

Data for the first five, the fastest, extrema, have the greatest measurement errors. The confidence intervals for the means were obtained using 15-min intervals of $f_{0} \mathrm{~F} 2$ measurements. The confidence intervals for extrema from 1 to 3 were calculated from the data of the second station, and for all others extrema from the first station. The ionosonde measurement time is about $1 \mathrm{~min}$, the data were read from a small graph which was obtained by data averaging over an unknown number of explosions. As noted, data from 27 explosions were analyzed, but how many of them were in the range from 20 to $30 \mathrm{Mt}$ are not specified in the article. We believe that the confidence intervals calculated for the 15-min sampling intervals are only a part of the uncertainty interval. The error of the experimental data relative to the model values is $25.8 \%$. On the whole, these data confirm the validity of the $V_{i}$ model.

On panel 2, the first 11 extrema of the model are compared with those obtained after a nuclear explosion $(Q=58 \mathrm{Mt}$ TNT) over Novaya Zemlya, detonated by the Soviet Union, at 30/10/ $196108: 33$ UT at altitude $z_{\text {expl }}=3.7 \mathrm{~km}$ (Row 1967). In this experiment, the critical frequencies $f_{0} \mathrm{~F} 2$ were measured by ionosondes at several stations in $15 \mathrm{~min}$ intervals. The stations were located at ranges $1250-4400 \mathrm{~km}$ from the explosions. Despite the enormous nuclear yield, the 15-min sampling rate allowed to record only the extrema four to eleven. The minima, i.e., extrema with numbers 6,8 , and 10 , were determined by interpolation using a ninth order regression. Omission of the first three extrema can be easily explained by the following considerations. In accordance with the $V_{i}$ model, the first extremum should be detected by the ionosondes closest to the source Kiruna $\left(R_{S}=1250 \mathrm{~km}\right)$ and Lycksele $\left(R_{S}=1650 \mathrm{~km}\right)$, only in $6.9 \mathrm{~min}$ and $9.2 \mathrm{~min}$, respectively, but it could not be recorded since the first measurement was made only $12 \mathrm{~min}$ after the explosion. The next two extrema (the duration of their existence over the measuring station was probably less than a minute) have also been missed owing to the large sampling period. Therefore, the first registered disturbance is actually a fourth extremum (minimum). Taking into account these limitations and the measurement errors we may conclude that the model and experimental curves are in good agreement, and the average error is $13.9 \%$. Without such correction the difference between the experimental data and the $V_{i}$ model could not be explained by any model of Earth's atmosphere. The confidence intervals were calculated for the Uppsala station $\left(R_{S}=2123 \mathrm{~km}\right)$ which is about the average distance from the explosion center to measuring stations.

Panel 3 shows the data collected by a network of groundbased ionosondes (located in Pacific Islands) after a megaton low-altitude US nuclear explosion in Johnston Island area on 30/10/1962 at 16:02 UT (Albee \& Kanellakos 1968). Ground stations were located at $R_{S}=1000-6500 \mathrm{~km}$.

The first disturbance recorded in this experiment corresponds to a transition through zero of the AGW train and before the second maximum (third extremum), predicted by the model. According to our analysis, missing the first two extrema in this experiment cannot be explained by the measurement resolution (equal to $5 \mathrm{~min}$ ), but by orientation of the wave vector of the first AGW half-period relative to the geomagnetic field lines at the measuring stations. The first half-period is classified as an LS AGW whose wave vector is parallel to the Earth's surface. Magnetic inclination angles for all measuring stations were in the range $30-40^{\circ}$, and vertical oscillations in transverse AGW are practically orthogonal to the magnetic field lines. Only in subsequent train extrema, propagating in free-wave mode, the wave vector after reflection from the Earth's surface is oriented so as to allow plasma oscillations along the lines of force. Taking these considerations into account the experimental data error is $5.3 \%$.

The first TID maximum following the volcano Pinatubo explosive eruption, recorded 15/06/1991 near Japan $\left(R_{S}=3000 \mathrm{~km}\right)$ (Igarashi et al. 1994), corresponds to the seventh extremum predicted by the model. Since the velocities of this and subsequent extrema are small (see Table 1), the measurement in 15-min intervals of the apparent reflection height of the F2-layer provided data for comparison with the model which was proven to be in error by $4.4 \%$ (see panel 4 in Fig. 7a)

Comparison of data predicted by the $V_{i}$ model and experimental data collected after five high-altitude nuclear explosions over Johnston Island in $1962\left(z_{\text {expl }}\right.$ varied from several dozens to $400 \mathrm{~km}$ ) is presented in Figure $7 \mathrm{~b}$. The experiment involved more than 60 ionospheric stations $\left(R_{S}\right.$ was in the range 1000 $14000 \mathrm{~km})$.

In panels 1-3 the data from the studies of Lomax \& Nielson (1968) and Breitling \& Kupferman (1967) are combined. These data were collected for the same nuclear explosions at ranges of $\sim 1000-5000 \mathrm{~km}$ and 4000-14 $000 \mathrm{~km}$ with different temporal discretization equal to $1 \mathrm{~min}$ in the first work and $1 \mathrm{~h}$ in the second. Confidence intervals for data were estimated at $R_{S}=2000 \mathrm{~km}$ and $R_{S}=8000 \mathrm{~km}$, which correspond to the mean distances for each measuring station involved in the two studies. The considerable spread of the confidence intervals can be explained by the different time sampling for the data.

Panel 1 shows the results obtained after an explosion with $Q=1.4$ Mt TNT on 09/07/1962 at 09:00 UT at the height $z_{\text {expl }}=399 \mathrm{~km}$. The data errors for the minute and hour discretization are $34 \%$ and $79 \%$, respectively. The maximum $R_{S}$ was approximately $14000 \mathrm{~km}$. The results from a nuclear test $(Q=0.02$ Mt TNT) performed 20/10/1962 at 08:30 UT at the height $z_{\text {expl }}=147 \mathrm{~km}$ are shown in panel 2. Here, the data errors are $14 \%$ and $56 \%$, respectively. The dependence of the extremum velocity on its number in the TID train for a third 


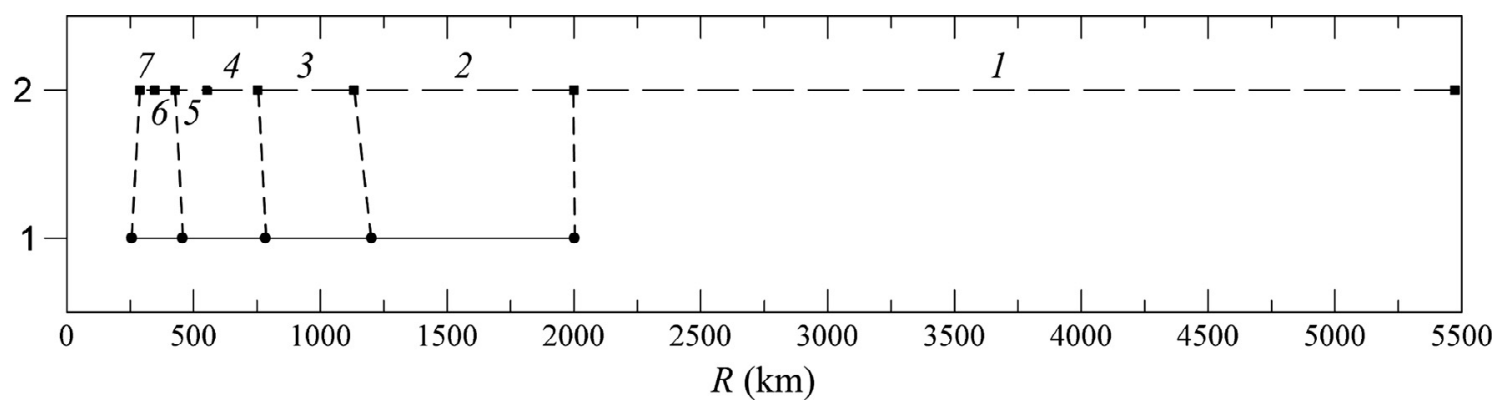

Fig. 8. Scans of AGW train half-periods. 1 - experimental values (Albee \& Kanellakos 1968), 2 - values predicted by the model.

Table 5. Velocities $V_{0 i}$ versus extremum index $i$.

\begin{tabular}{lc}
\hline \hline$i_{0}$ & $V_{0 i}\left(\mathrm{~km} \mathrm{~s}^{-1}\right)$ \\
\hline 1 & 1.7500 \\
2 & 0.8540 \\
3 & 0.5618 \\
4 & 0.4041 \\
5 & 0.3137 \\
6 & 0.2593 \\
7 & 0.2176 \\
8 & 0.1858 \\
9 & 0.1660 \\
10 & 0.1513 \\
11 & 0.1328 \\
12 & 0.1148 \\
13 & 0.1017 \\
14 & 0.0820 \\
\hline
\end{tabular}

nuclear test $(Q=0.41 \mathrm{Mt}$ TNT) on $01 / 11 / 1962$ at $12: 10 \mathrm{UT}$ at $z_{\text {expl }}=97.5 \mathrm{~km}$ is illustrated in panel 3 . The data errors are $25 \%$ and $34 \%$, respectively. In the last two experiments, the maximum value of $R_{S}$ was $\sim 12500 \mathrm{~km}$.

Panels 4 and 5 show the results calculated using the data in Breitling \& Kupferman (1967) for nuclear explosions performed 26/10/1962 at 10:00 UT and 04/11/1962 at 07:30 UT at the height of a few tens of kilometers. The nuclear yields were $\approx 0.2-1 \mathrm{Mt}$ TNT and $0.02 \mathrm{Mt} \mathrm{TNT}$, respectively. The data errors of the experimental results for these explosions are $28 \%$ and $20 \%$, respectively. The confidence intervals were calculated for $R_{S}=8000 \mathrm{~km}$, and the maximal value of $R_{S}$ was about $12500 \mathrm{~km}$.

The experimental data errors relative to the data predicted by the $V_{i}$ model for near-surface and high-altitude nuclear explosions are approximately the same. Errors assigned to the data from Breitling \& Kupferman (1967) are not taken into account in this comparison because to their large errors due to the 1-h discretization. Our conclusions were obtained using only data with approximately the same measurement error (measurement interval equal to $15 \mathrm{~min}$ and less). In general, we claim that the results of the above experiments confirm the $V_{i}$ model adequately.

\subsection{The dynamics of horizontal spatial half-periods}

A spatial train position, consisting of four half-periods defined by zero values of the disturbance, was given in the work by Albee \& Kanellakos (1968) Observational data were obtained after a US megaton nuclear detonation near the Earth's surface on $30 / 10 / 1962$. We used these data to test the model validity.
Figure 8 shows the spatial position of the observed (1) and modeled (2) trains of spatial half-periods. As noted above, the first two extrema were missed in these nuclear tests, and the measurements start at zero perturbation (the second in the train), located in front of the third extremum (second maximum). Thus, the experimental train starts from the second half-period.

Table 1 shows propagation velocities $V_{i}(i)$ of the first 15 extrema ( $i$ is extremum index). We obtained the velocity of AGW train fragments located between each pair of adjacent extrema and the corresponding unperturbed values of air pressure fluctuations in the train using tenth regression of $V_{i}(i)$. Table 5 shows the values of phase velocities of the train fragment $V_{0 i}\left(i_{0}\right.$ is the index of undisturbed area in the train). Calculation of spatial half-periods was carried out using relation (3), where the velocities $V_{i}$ were substituted by $V_{0 i}$ (see Table 5).

The second and third half-periods predicted by the model coincide practically with corresponding experimental data as far as the values and positions relative to the disturbance source are concerned. The half-period indexing goes from right to left and is performed using the model half-period marked by the markers from 1 to 7 in upper part of Figure 8. Experimental extrema observed by Albee \& Kanellakos (1968) are connected by quasi-vertical dashed lines with extrema predicted by our model. According to the figures with experimental data $\Delta N /$ $N(t)$ given in the article by Albee \& Kanellakos (1968), only the first three extrema, corresponding to the third, fourth, and fifth extrema predicted by the model, are clearly marked, since their amplitudes $A_{d}$ reach $50-100 \%$, while $A_{d}$ for the subsequent extrema are $\sim 15$ times smaller and are close to natural background fluctuations.

Therefore, only the second and third experimental half-periods are indubitable. We suppose that the last two experimental half-periods shown in the Figure 8 are really combination of the fourth and fifth, sixth and seventh quasi-periods. This is evidenced by the amplitude and spatial location of these TID fragments relative to the source. The last experimental half-period could also be the sum of the 6 th, $7^{\text {th }}$, and 8 th half-periods, since the 8 th model half-period is very small and is compared with marker dimensions.

These assumptions are confirmed in Figure 9, which presents the experimental (curve 1) and model (curve 2) dependences of the half-period lengths upon the distance to the disturbance center. Experimental and model values for the second and third half-periods are practically the same, while those for the fourth and fifth half-periods are not as close as the others, due to the growth of experimental errors. The model error for the second and third half-periods is about $6.5 \%$. 
Y.P. Fedorenko et al.: Model Traveling Ionospheric Disturbances

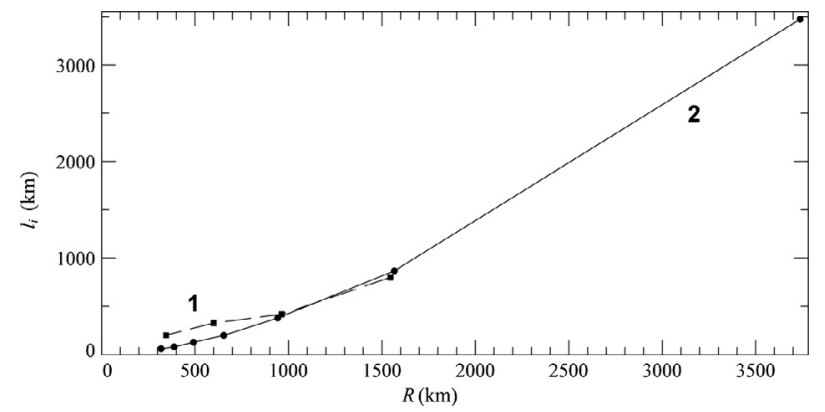

Fig. 9. Lengths of half-periods versus distance between their centers and a source. 1 - experimental values (Albee \& Kanellakos 1968); 2 - values predicted by the model.

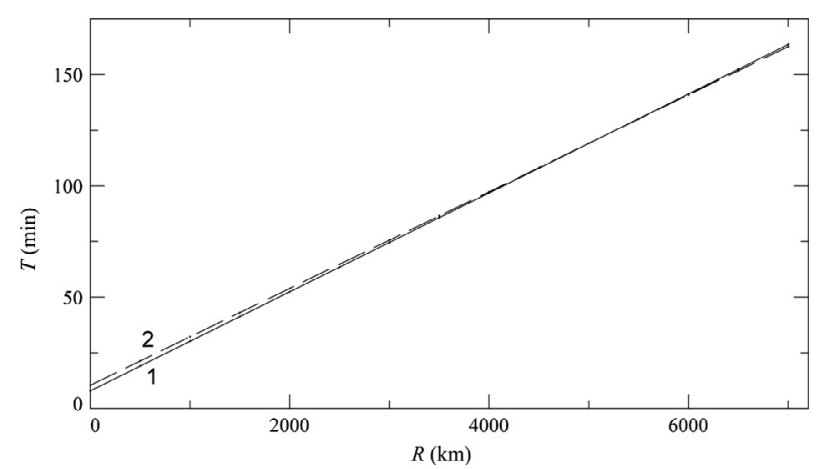

Fig. 10. Temporal periods versus a distance $R$ to the source. $1-$ values predicted by the model; 2 - experimental values (Albee \& Kanellakos 1968).

\subsection{The dependence of the TID temporal period upon the distance from the source}

The relation between the first measured temporal period and the distance to the explosion, carried out by the US 10/30/1962, can be found in Albee \& Kanellakos (1968) (see curve 2 in our Fig. 10). As stated above, the first and second extrema were missed in this experiment, and the first recorded period corresponds to the sum of the third and fourth half-periods. The starting point of the first half-period is the transition through zero $\Delta N_{m}$ between the second and third model extrema. Therefore, we compared the experimental curve with the model curve, which is the sum of the third and fourth half-periods (curve 1). The average error of the model curve does not exceed $3.6 \%$. For the same nuclear test we compare in Figure 11 the model (curve 1) and experimental (curves 2) spatial dependencies for the third (panel a) and fourth (panel b) half-periods. The experimental curves are the first-order regression of the experimental values shown in the graph by square markers. The average errors of the model dependence relative to the regression of experimental values for the third and fourth half-periods are 7\% and $12 \%$, respectively.

In the works by Row (1967) and Roberts et al. (1982a) the experimental data obtained from a nuclear test in Novaya Zemlya 30/10/1961 and from the St. Helens volcano explosion $18 / 05 / 1980$ are presented. In these studies, they reported that 10 to 11 extrema were recorded in the TID trains for different distances from the disturbance sources. In Figure 12 we compare the spatial dependence of the average time period revealed in these experiments and two times the model half-periods averaged over the first ten half-periods (curve 1). Curve 2 presents
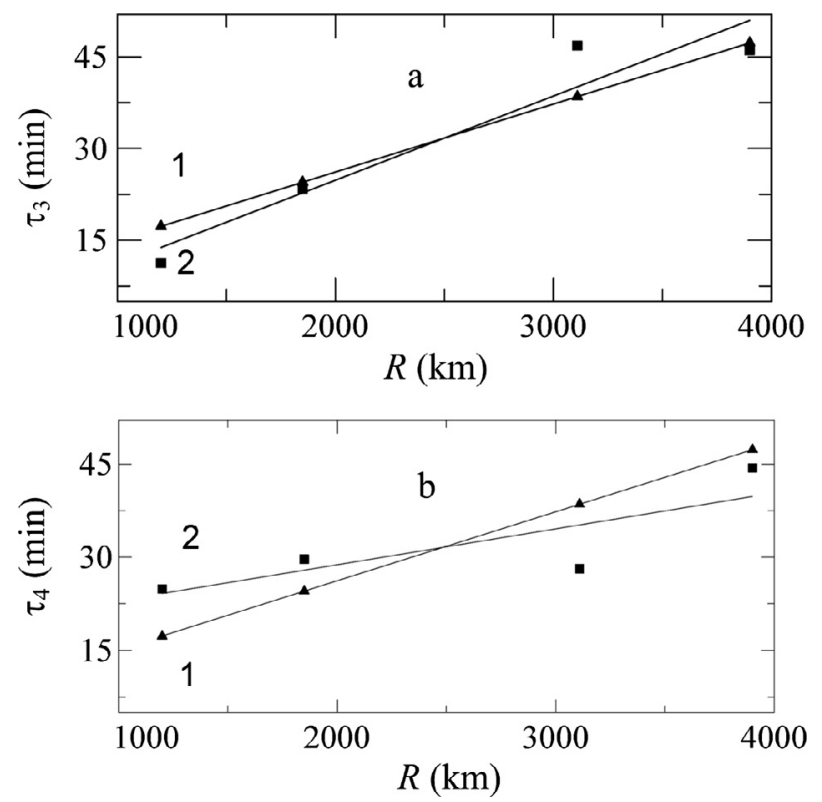

Fig. 11. The third (panel a) and fourth (panel b) temporal halfperiods versus a distance $R$ to the source. 1 - values predicted by the model; 2 - experimental values (Albee \& Kanellakos 1968).

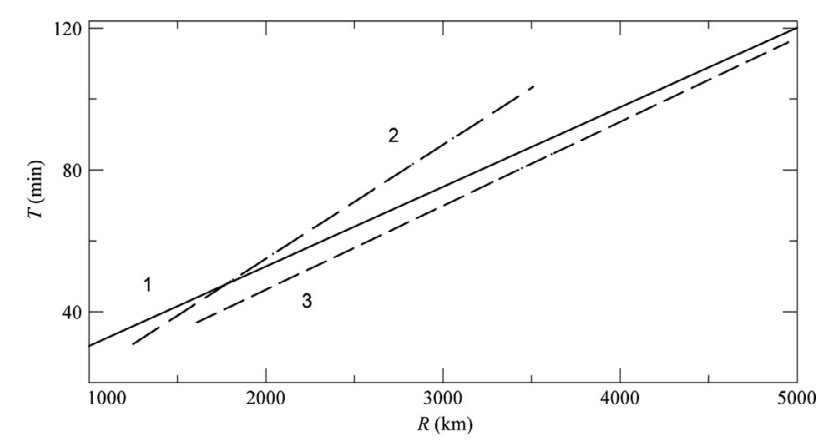

Fig. 12. Temporal period $T$ versus distance $R$ to the source $1-$ values predicted by the model; 2 - Row (1967); 3 - Roberts et al. (1982a).

the dynamics of TID quasi-periods registered after the nuclear explosion performed by the USSR at Novaya Zemlya (Row 1967).

Curve 3 is the average temporal period in the AGW train, obtained by Roberts et al. (1982a) after the St. Helens volcano explosion. The average error of the model (curve 1) with respect to the experimental curves (curves 2 and 3 ) is $10 \%$.

\subsection{Electron density decrease observed in connection with the propagation of a TID train}

Lomax \& Nielson (1968), analyzing the effects of three highaltitude nuclear tests, discovered an unexplained effect of marked decrease in electron density along the propagation path of a TID train within an area of $2500-3000 \mathrm{~km}$ radius. The effect was detected using vertical and oblique sounding with 1-min resolution, which significantly improved the data quality, as compared to earlier experiments conducted with sampling periods of $1 \mathrm{~h}, 15 \mathrm{~min}$, and rarely $5 \mathrm{~min}$. The measurement of total electron content by satellite beacons, made 12 and $15 \mathrm{~h}$ after the explosion, revealed a TEC reduction up to $30 \%$ as compared to TEC measured the day before. The nuclear 
tests were performed at night on $09 / 07 / 1962(Q=1.4 \mathrm{Mt}$ TNT, $\left.z_{\text {expl }}=399 \mathrm{~km}\right), \quad 20 / 10 / 1962 \quad(Q=0.02 \quad$ Mt TNT, $\left.z_{\text {expl }}=147 \mathrm{~km}\right)$, and $01 / 11 / 1962(Q=0.41$ Mt TNT, $\left.z_{\text {expl }}=97.5 \mathrm{~km}\right)$.

According to our AGW-TID model the propagation velocity of the zero extremum (minimum) in the train is more than $11.3 \mathrm{~km} \mathrm{~s}^{-1}$, its amplitude $A_{0}=0.48$. Therefore, at a distance of $3,390 \mathrm{~km}$ from the explosion center the atmospheric pressure will be much lower than the natural background value $5 \mathrm{~min}$ after the explosion. Since the lifetime of disturbances in the ionospheric F-region can be more than $10 \mathrm{~h}$ (Tyrnov 2005), a long living area of reduced electron density values with a spatial extent of about $7000 \mathrm{~km}$ is formed. As noted above, the second extremum with amplitude $A_{i}=0.83$ (first peak), propagating at $3 \mathrm{~km} \mathrm{~s}^{-1}$, was not recorded in these experiments since air oscillations in AGW were orthogonal to the magnetic field lines. For the St. Helens volcano eruption (Roberts et al. 1982a), the registration conditions were more favorable in this respect, and the first extremum as well as the onset and subsequent extrema were registered.

We now return to the results of high-altitude nuclear tests. The extrema of AGW trains having smaller velocities and propagating in an area with reduced density develop the next slower TID train extrema. Thus, at distances ranging from 1000 to $5000 \mathrm{~km}$ the measurement results are a superposition of longlived disturbance phases and oscillations with temporal periods from 30 to $120 \mathrm{~min}$ (see also Fig. 12). We believe that the above-mentioned unexplained effect indicates that the onset has already passed and gave rise to a region of lower concentration (also called a hole), which may be long living due to low solar activity. Such regions are formed both during nuclear tests (e.g. Lomax \& Nielson 1968) and energetic particles precipitation in the magnetospheric cusp after sudden commencement of magnetic storm (Shashunkina 1966, 1968a, 1968b, 1972).

A similar signature of a low electron density region was observed after a nuclear explosion at Novaya Zemlya on 30/ $10 / 1961\left(Q=58\right.$ Mt TNT, $\left.z_{\text {expl }}=3.7 \mathrm{~km}\right)$. Adushkin \& Gorelyi (1994) give results in graphical form of the F2-layer critical frequency $\left(f_{0} \mathrm{~F} 2\right)$ measurement, made in the period from $27 / 09 / 1961$ to $05 / 10 / 1961$ at the following ionospheric stations: midlatitude Alma-Ata (USSR, $R_{S}=3500 \mathrm{~km}$ ), southern hemisphere Mundaring (Australia, $R_{S}=12000 \mathrm{~km}$ ), and high-latitude Mirny (Antarctic, $R_{S}=16000 \mathrm{~km}$ ). The measurements began $100 \mathrm{~h}$ before the explosion and continued for up to $200 \mathrm{~h}$ after the explosion. The critical frequencies $f_{0} \mathrm{~F} 2$ above Alma-Ata were reduced for $44 \mathrm{~h}$, and then began to increase, while above the Southern Hemisphere sites they were enhanced for about $50 \mathrm{~h}$ at Mundaring and about $59 \mathrm{~h}$ at Mirny and then began to decrease. The maximal amplitude (about $50 \%$ ) of this long-period wave was observed over Mundaring. Data presented in the paper do not allow us to calculate the wave velocity due to small plot scale, but we can assert that the concentration began decreasing over Alma-Ata $17 \mathrm{~h}$ after explosions, and began increasing after $21 \mathrm{~h}$ over Mundaring and after $35 \mathrm{~h}$ over Mirny. The experimental results presented in that paper indicate that if an AGW propagates from north to south in the northern hemisphere, AGW air pressure minima generate TID minima, and in the southern hemisphere, AWG minima generate TID maxima. Moreover, the duration of TID half-periods is increasing while the TID moves away from the explosion epicenter. These two effects confirm that our model is valid at distances up to $16000 \mathrm{~km}$ from the disturbance source.

\subsection{Global observation of AGW and TID}

Disturbances generated by the nuclear tests on Novaya Zemlya on $23 / 10 / 1961(Q=25 \mathrm{Mt}$ TNT$)$ and 30/10/1961 $(Q=58 \mathrm{Mt}$ TNT, $\left.z_{\text {expl }}=3.7 \mathrm{~km}\right)$ are the most studied globally, since atmospheric pressure waves propagated across the globe. For the second event pressure disturbances were observed by all ground stations. Isolines of disturbance delay time in both hemispheres were similar to concentric circles centered at the explosion epicenter (Wexler \& Hass 1962). Using high-quality micro-barograms recorded in Washington at $R_{S}=7000 \mathrm{~km}$ (Wexler \& Hass 1962), we estimated that the velocities of pressure waves at the Earth's surface for these two events were equal to 0.33 and $0.31 \mathrm{~km} \mathrm{~s}^{-1}$. The velocity of the pressure wave generated by the second event, averaged over the globe as reported by Wexler \& Hass (1962) and Obayashi (1962), was $0.31 \mathrm{~km} \mathrm{~s}^{-1}$.

However, ionospheric disturbances were not observed by all stations. Ionospheric disturbances generated by the two events were observed in Europe, Asia, India, and Southeast Asia. Small disturbances were observed in Japan, and only very minor disturbances or no disturbances at all were observed in North America (Obayashi 1962), despite the fact that $R_{S}$ for stations in North America are comparable with $R_{S}$ for the European stations. These dead zones of the ionospheric disturbances confirm the validity of our model, which postulates that the ionospheric manifestation of atmospheric waves is determined by the angle between the wave vector of AGW and the geomagnetic meridian plane, and by the projection of the ionized component velocity on the geomagnetic field vector. Possible distortions of the AGW front, caused by regional wind velocities and by pressure and temperature heterogeneities, observed, for example, by Wexler \& Hass (1962) should be also taken into account. As it turned out, the front curvature of the direct wave can reach $40^{\circ}$.

\section{Model validation by ionosphere sounding using signals of low-orbit satellites}

\subsection{The research methodology}

Spatial distributions of the electron density were obtained from radio signals radiated by the navigational satellites Cicada and Cicada-M (Russia) orbiting at an altitude of approximately $1000 \mathrm{~km}$ at an inclination of $83^{\circ}$. The onboard transmitter continuously radiates coherently at 150 and $400 \mathrm{MHz}$. A ground station located at the Radiophysical Observatory of V.N. Karazin Kharkiv National University $\left(\varphi=49.63^{\circ} \mathrm{N}, \lambda=36.32^{\circ} \mathrm{E}\right)$ receives the signals and determines the ionospheric component of the Doppler shift $\delta f_{i}$ for 12-16 min every 1.5-2 h. Registrations of $\delta f_{i}(t)$ are indicators of the state of the ionosphere, allowing to investigate the regular and perturbed parameters of the spatial distribution of electron density for a region in the latitude range $33-66^{\circ} \mathrm{N}$, corresponding to $\sim 3600 \mathrm{~km}$ at the Earth's surface. During a single satellite pass, the ionosphere parameters can be inferred over a latitude range of $24^{\circ}$ (about $2700 \mathrm{~km}$ ). Details of the method are described by Tyrnov et al. (2005) and Fedorenko et al. (2010b, 2012).

\subsection{TID occurrence}

We have analyzed the dependency of $\delta f_{i}(t)$, recorded in 1987 2010 under specific heliogeophysical conditions. The amplitude of the effects caused by the TIDs is ten to hundred times greater 
Y.P. Fedorenko et al.: Model Traveling Ionospheric Disturbances

Table 6. TID occurrence.

\begin{tabular}{|c|c|c|c|c|c|c|c|}
\hline \multirow[t]{2}{*}{ Solar activity cycle } & \multirow{2}{*}{$\begin{array}{c}\text { Total number of } \\
\text { observation }\end{array}$} & \multicolumn{2}{|c|}{ Winter } & \multicolumn{2}{|c|}{ Summer } & \multicolumn{2}{|c|}{ Equinox } \\
\hline & & $\begin{array}{l}\text { Sample } \\
\text { size }\end{array}$ & Occurrence & $\begin{array}{l}\text { Sample } \\
\text { size }\end{array}$ & Occurrence & $\begin{array}{l}\text { Sample } \\
\text { size }\end{array}$ & Occurrence \\
\hline $\begin{array}{l}\text { 1997-1999 Moderate } \\
\text { activity }\end{array}$ & 647 & 158 & 0.89 & 185 & 0.86 & 304 & 0.79 \\
\hline 2009-2010 Low activity & 604 & 79 & 0.98 & 235 & 0.97 & 290 & 0.98 \\
\hline
\end{tabular}

than the instrumental error $\delta=0.005 \mathrm{~Hz}$. The high measurement accuracy and huge amount of data, accumulated over two cycles of solar activity, allow us to disprove conclusions of other researchers, who conducted experiments with the low-orbit navigation satellite NNS (Transit) and NNSS (USA) but with insufficient measurement accuracy. According to our observations, the generally accepted view concerning the presence of latitudinal, seasonal, and diurnal occurrence of MS TID is not true. For example, Evans et al. (1983) and Ogawa et al. (1988) state that MS TIDs are observed least often in the night, and in the summer there is a minimum of their occurrence. We have analyzed the data provided in the above-cited papers, and concluded that the results may be explained by the lack of the measurement precision. There exists a threshold value of minimum electron density $N_{m}$, below which the measurements are impossible. It should be noted that both the above-cited papers make similar statements. Evans et al. (1983) claim that the poor measurement accuracy influences only the amplitude of detected dependencies and does not affect their validity: "... which we believe are attributable, in part, to the diurnal and seasonal variation in total electron content, together with a minimum size detectable disturbance". As far as diurnal occurrence is concerned, Ogawa et al. (1988) state: “... which may result from easier detectability of TID's during daytime hours". Observations described in these studies were carried out in different hemispheres, so when Evans et al. (1983) have observed a maximum in the winter, Ogawa et al. (1988) have observed a summer minimum at the same time (and vice versa). According to our hypothesis, most TIDs of natural origin are generated by energetic particle precipitation in the magnetospheric cusp, therefore, the TID occurrence should not depend upon the seasons. Moreover, even a single act of AGW excitation generates TIDs observed almost for $24 \mathrm{~h}$. Therefore, maxima and minima in the diurnal and seasonal dependence of the TID occurrence would be observed if the measurement accuracy is low.

Tsugawa et al. (2007a, 2007b); Kotake et al. (2007); Song et al. (2011); Otsuka et al. (2013) and Vlasov et al. (2011) after almost three decades of research performed by Evans et al. (1983) and Ogawa et al. (1988) have also studied the diurnal and seasonal dependence of TID occurrence. However, the dependences obtained by these authors contradict each other and previous results. For example, Tsugawa et al. (2007b) observed minimum occurrence in the summer 2006 while Vlasov et al. (2011) detected maxima in the summers 2007-2008. Vlasov deduced that a seasonal dependence of TID amplitude was absent and that MS TIDs are not observed in near midnight hours. These findings are consistent with our results. Differing conclusions regarding diurnal and seasonal occurrence may be explained by measurement errors of AGW-TID parameters, which were determined by measuring the TEC, electron density, ion velocity, electron and ion temperatures. A possible cause of such dependence is explained by Vlasov et al. (2011) as follows: "The seasonal variation in AGW-TID occurrence achieved in this study follows closely the seasonal variation of ionospheric electron content at the observing site. It remains still as a topic for future studies whether the observed seasonal variation is natural or caused by seasonal variation in the performance of our observation method"; "The AGW signatures are clearest in ion velocity data. One has to remember that if we do not see the signatures in the other ISR parameters it does not mean their total absence. For example, in electron density the AGW signatures can be masked by some other background processes".

Continuous registration of $\delta f_{i}(t)$ allowed us to diagnose the ionosphere for all seasons on a 24-h basis for different levels of solar and geomagnetic activity. We have determined that TID occurrence does not depend on the time of day. Table 6 shows the seasonal TID occurrence during the years of moderate and low solar activity. Following the paper of Evans et al. (1983), we classify measurements made from October 27 to February 4, from April 30 to August 2, from February 5 to April 29, and from August 3 to October 26 as winter, summer, and equinox measurements, respectively. Figure 13 illustrates the annual average of TID occurrence (circles) and its linear regression (curve 1), the yearly average of sunspot number (curve 2), and annual average of TID occurrence with linear trend removed (curve 3) for the period from 1987 to 2010 .

Based on the above analysis we conclude that during the 23-year period the TID occurrence does not depend on the season and on sunspot number. The TID occurrence, defined as ratio of TID events to the total number of observations for the corresponding period, increased from $\sim 50 \%$ in 1987 to $\sim 98 \%$ in 2010 , and the average was $87 \%$. This conclusion may explain the scatter of occurrence values observed during the 60 -year period. At the same time, we support the conclusion of most researchers that MS TID occurrence does not depend on the magnetic activity (e.g., Evans et al. 1983; Ogawa et al. 1988; Hocke \& Schlegel 1996; Tyrnov et al. 2005; Tsugama et al. 2007a). In the years of minimum solar activity, about $70 \%$ of LS TIDs are observed in the midnight time and about $30 \%$ in the morning.

In addition, we managed to explain latitudinal dependence of MS TID occurrence and reasons why MS TIDs are simultaneously detected both on the northern and southern branches of the $\delta f_{i}(t)$ registrations. It turns out that TIDs exist in the entire observation area and are detected at one or both $\delta f_{i}(t)$ branches, depending on the inclination angle of the TID front in a vertical plane (Fedorenko et al. 2010b). This effect will be described in detail below.

We use this effect to classify TIDs by their propagation mode, namely, the waveguide mode and free-wave mode. According to our model, the LS TIDs propagate only in waveguide mode, while MS TIDs can propagate in both modes. If the spatial horizontal period of MS TID does not depend on the longitude, we assume that the TID propagates in the waveguide mode. If MS TIDs were observed only in the northern or 

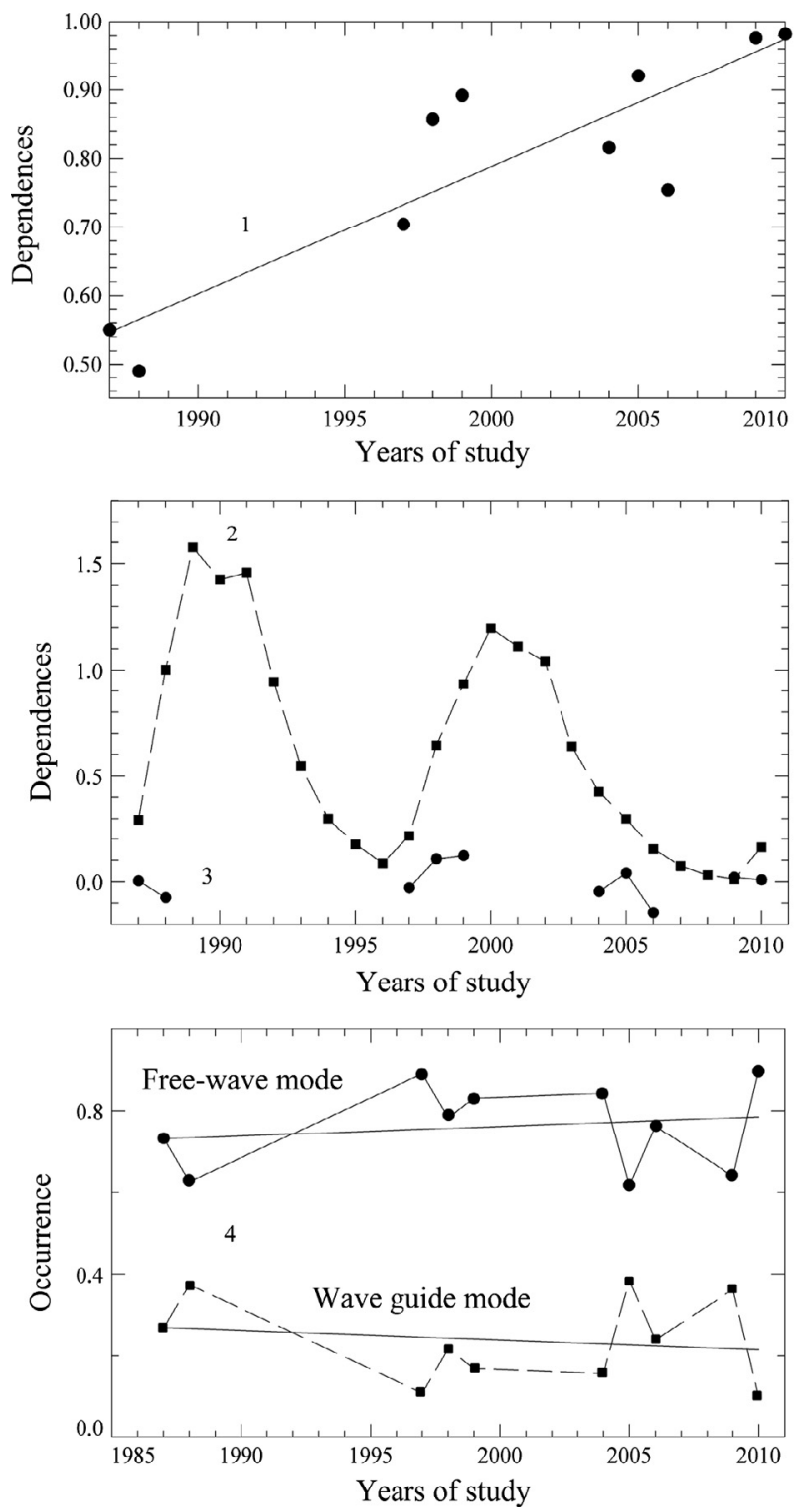

Fig. 13. TID occurrence and solar spot numbers in 1987-2010: 1 linear regression of TID occurrence; $2-\mathrm{W} / 100$, W is yearly average Wolf number; 3 - TID occurrence without a linear trend, 4 - the occurrence of TIDs propagating in waveguide and free-wave modes and their linear regressions for the period from 1997 to 2010 .

southern part of the area where $\delta f_{i}(\varphi)$ was recorded or their amplitudes are dominated in one of these parts, we assume that a free-wave mode was present. The distribution of the TID with respect to the propagation mode was carried out according to the prevailing influence of one or another TID type on the registration of $\delta f_{i}(t)$. Therefore, if an MS TID and an LS TID are simultaneously traced in a $\delta f_{i}(t)$ registration under the conditions that LS TID only slightly deforms registration obtained under undisturbed conditions, the TID is classified as propagating in free-wave mode. We analyzed only the registrations in which such classification can be reliably made. As a result of this selection we retained for periods 1977-1999 and 20092010 only about $50 \%$ and $30 \%$, respectively, of the available registrations. The analysis results are shown in Table 7.

As can be seen, the occurrence of TIDs propagating in a free-wave mode (MS TIDs only) is from 3 to 18 times greater than that for TIDs propagating in a waveguide mode. We did

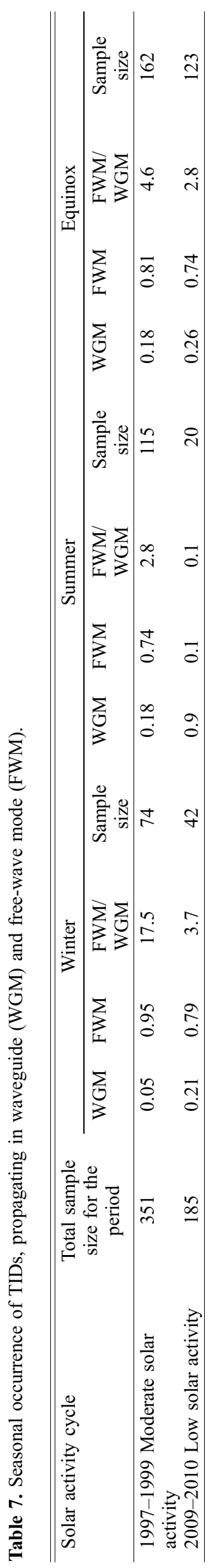


not take into account the summer data for the period from 2009 to 2010 when the ratio WGM/FWG was equal to 0.1 , for yet unexplained reasons, which are anomalous as far as the propagation mechanism of natural TID is concerned. Perhaps it may be explained by small statistics for the selected period. Overall, the results confirm the well-known conclusion that LS TIDs are observed much less frequently than MS TID. We will show below that an LS TID may exist for several hours and MS TIDs of different scales can propagate against their background. These data are consistent with the dynamics of TID and its horizontal dimensions as described by our model, which predicts that an initial disturbance generates high-speed LS TID, followed by MS TID propagating for a much longer time period with a much lower velocity.

At the bottom panel of Figure 13 the occurrence of TIDs propagating in waveguide and free-wave modes and their linear regressions for the period from 1997 to 2010 are shown. No dependence on sunspot number was detected. The regression lines are nearly horizontal, suggesting no epoch or solar cycle dependence. According to the data for this period the TID occurrence propagating in free-wave and waveguide mode is $76 \%$ and $24 \%$, respectively. In fact, the statistics reflects the observation time of MS and LS TID. Thus, MS TIDs are observed three times more often than LS TIDs.

\subsection{The minimum values of TIDs horizontal scales}

As stated above, $L_{\text {min }}$ may vary in the range 200-747 $\mathrm{km}$ for $R_{S}$ between 500 and $3500 \mathrm{~km}$. Using cusp models, presented by Köhnlein \& Raitt (1977) and Deminov et al. (1992), we calculated that in our experiments registrations of $\delta f_{i}(t)$ start at distances $R \sim 500 \mathrm{~km}$ from the cusp. Therefore, the minimum value of $L$, calculated according to our model, should not exceed $200 \mathrm{~km}$. With a decrease of $R$ the value of $L_{\text {min }}$ is also reduced down to the minimum value of the selected train period, observed at its onset. We now estimate the value of $L_{\text {min }}$ for $R \sim 500 \mathrm{~km}$ in a slightly different way. According to our model, during initiation the AGW-TID spatial quasi-periods have the minimum values decreasing with their index in the TID train (see Table 2). We consider the quasi-periods which generate the MS AGW-TIDs at the given $R$. For example, the initial value for the seventh quasi-period $L_{\text {min }}=47 \mathrm{~km}$ linearly increases as the distance from the source grows. At a distance $R=480 \mathrm{~km}$ the value is $L=245 \mathrm{~km}$, and at $R=1022 \mathrm{~km}$ it is $L=412 \mathrm{~km}$. The first extrema are now at $R_{1}=1200 \mathrm{~km}$ and $20000 \mathrm{~km}$, respectively. For the fourth quasi-period, which was recorded much more frequently in our experiments, $L_{\text {min }}=104 \mathrm{~km}$ at $R=512 \mathrm{~km}$ and $R_{1}=7000 \mathrm{~km}, L$ predicted by the model is equal to $438 \mathrm{~km}$. At $R=1006 \mathrm{~km}$ and $R_{1}=11000 \mathrm{~km}$ the model value is $L=689 \mathrm{~km}$. Let us examine the panel 2 in Figure 4, which shows $L(R)$ for the trains in which the first extremum was at distances $R_{1}$ in the range from $4500 \mathrm{~km}$ to $8000 \mathrm{~km}$, corresponding to an elapsed time of 2544 min after the initiation of the disturbance. As can be seen from Figure 4, at a distance of $R=500 \mathrm{~km}$ the $L$ values for $R_{1}$ are in the range of $400 \mathrm{~km}$ to $600 \mathrm{~km}$. Thus, if MS TIDs dominate the ionosphere above a measuring station, the TID with periods less than $100-150 \mathrm{~km}$ should not be detected in the $\delta f_{i}(t)$ registrations.

We now turn to our observational data obtained under specific heliogeophysical conditions. As a result of our data analysis, we found that the observed minimum values of $L$ are greater than $\sim 150 \mathrm{~km}$. Similar conclusions were obtained by Francis (1975) and Fedorenko et al. (2010a, 2010b). The exper- imental verification of the lower bound for $L \sim 200 \mathrm{~km}$ validates our model adequacy.

\subsection{Precipitation of solar wind energetic particles into the magnetospheric cusp}

We suppose that the source of most disturbances observed during the whole period of our experimental studies is associated with the precipitation of solar wind energetic particles into the magnetospheric cusp of the northern hemisphere.

This statement may be confirmed by the following observations. The horizontal length $L$, the amplitude $A_{d}$, and the modulus of the inclination angle $\xi$ are increasing with decreasing latitude $\varphi$. Since $\xi$ is negative, the front TID in the vertical plane is tilted toward low $\varphi$ i.e., in the direction of AWG propagation. Now we will give separate experimental results supporting our conclusion.

Figure 14 shows $\delta f_{i}(\varphi)$ and $L(\varphi)$, obtained in winter, summer, and autumn, under average solar and geomagnetic activity $(W=52-97, K \mathrm{p}=3 \ldots 5+)$ around noon local time.

The $L$ values were determined by measuring the distance between adjacent minima or maxima $\delta f_{i}(\varphi)$, at various latitudes $\varphi$. Therefore, the number of recorded values is almost twice the number of TID quasi-periods appearing on the $\delta f_{i}(\varphi)$ registrations. The experiment date, the time of the satellite traverse $\left(t_{0}\right)$, the satellite zenith angle at the traverse $\left(\gamma_{0}\right), L, A_{d}, \xi$, and the unperturbed $N_{m}$ and $z_{m}$ over the measuring station at the time of measurement are shown in the plot. Hereinafter, we use the Standard Moscow Time (SMT), offset is UT +2 .

The above-mentioned parameters were obtained under the assumption that the TID wave vector is directed along a geographic meridian. The MS TIDs are clearly identified on the plots $\delta f_{i}(\varphi)$. It should be kept in mind that when a radio beam approaches the satellite traverse and the angle $\gamma_{0}$ is in a range $\sim 0^{\circ}-20^{\circ}$, the TID amplitude inferred from the measured $\delta f_{i}(\varphi)$ is significantly reduced even if the TID amplitude $A_{d}$ stays unchanged in the entire region of radio sounding (Tyrnov et al. 2005; Fedorenko et al. 2010b). Therefore, near the traverse our methodology is less suitable for TID detection. In addition, for large values of $\xi\left(>40^{\circ}\right)$ TIDs are observed only at that part of the $\delta f_{i}(\varphi)$ registration chain (before or after the traverse), which is further from the source (Fedorenko et al. 2010b). This effect specifies the direction of TID movement (south or north) and the approximate location of the source (to the south or north relative to a measuring station).

The half-width $H$ of the layer which guides the TID was $25 \mathrm{~km}$. We have found that for most irregularities the parameters are in the ranges: $A_{d}=0.07-0.17, L=250 \mathrm{~km}, \xi=42^{\circ}-$ $75^{\circ}$. For the most southern irregularities the corresponding values are: $A_{d}=0.13-0.5, L=415-600 \mathrm{~km}, \xi=59-75^{\circ}$. We calculated the above parameters using the model of perturbed spatial distribution $N(6)$. The parameters $N, N_{m}$, and $z_{m}$ of the unperturbed model were determined by adapting the global model of $N$ by the method described by Tyrnov et al. (2005). All $\delta f_{i}(\varphi)$ registrations indicate that TIDs always appear to the south of the latitude traverse, clearly indicating that the disturbance source was located northward of the measuring station.

The correlation coefficients of the linear regression $L(\varphi)$ were in the range from 0.78 to 0.99 . Figure 4 shows the approximately linear increase in spatial quasi-periods $L$ of a TID train as predicted by our model when the wave packet moves away from the source. Here we present the model dependence of 

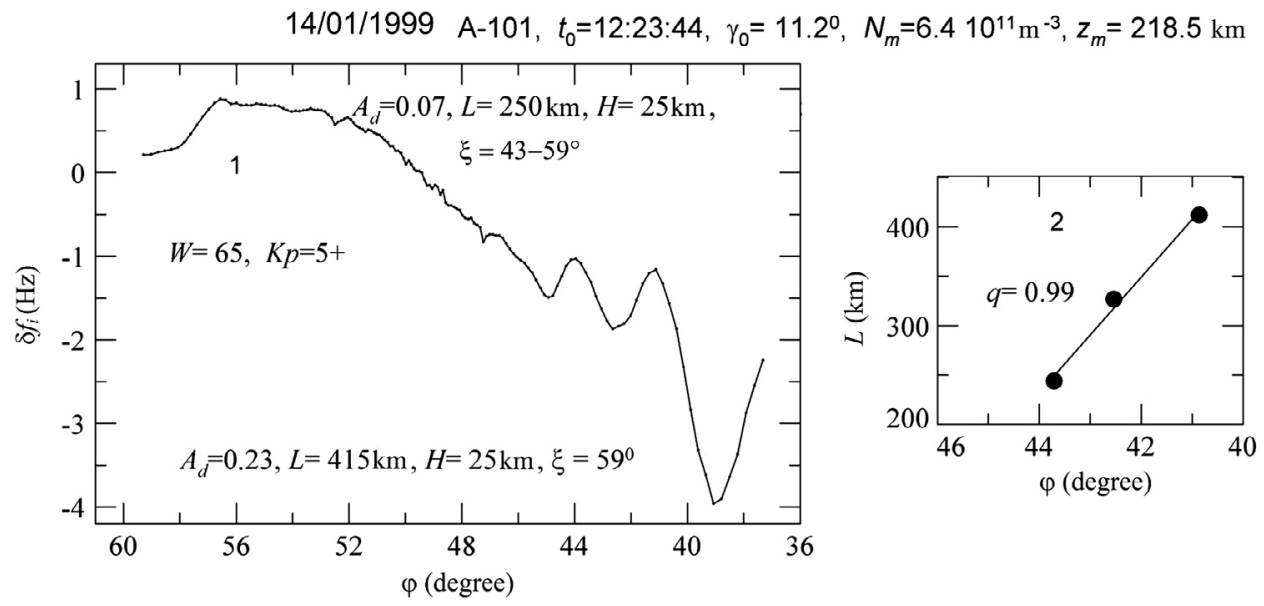

23/07/1999 A-101, $t_{0}=14: 30: 49, \gamma_{0}=4.5^{0}, N_{m}=7.210^{11} \mathrm{~m}^{-3}, z_{m}=321.5 \mathrm{~km}$
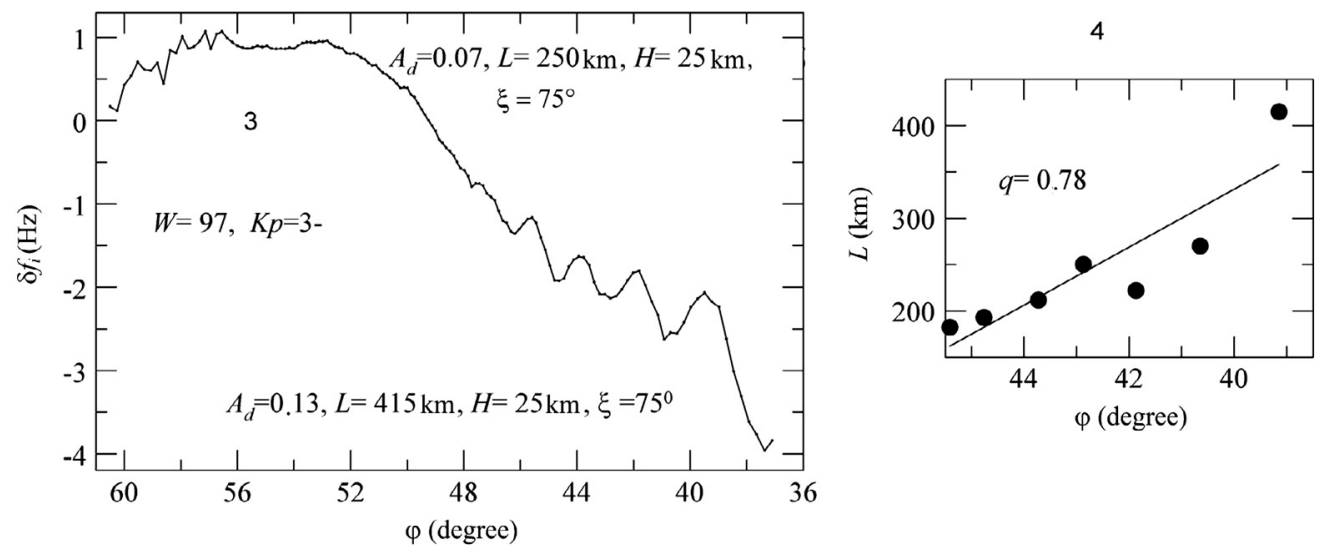

09/11/2004 A-102, $t_{0}=10: 44: 47, \gamma_{0}=27.9^{0}, N_{m}=5.510^{11} \mathrm{~m}^{-3}, z_{m}=198 \mathrm{~km}$,
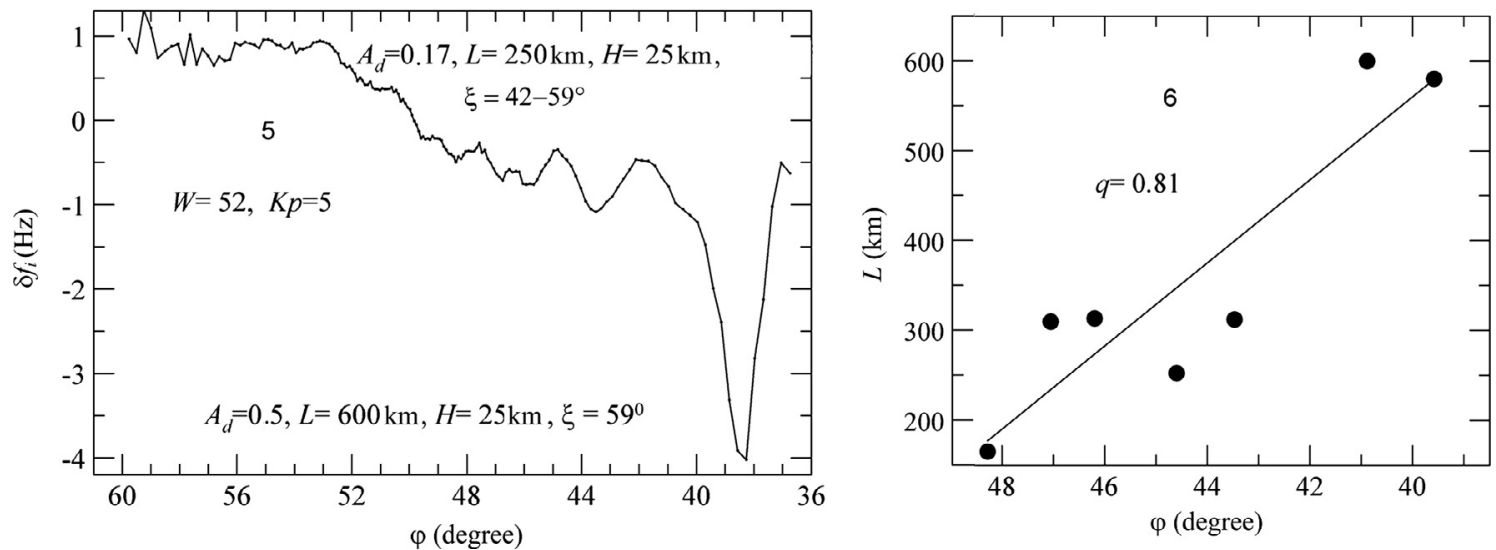

Fig. 14. The results of MS TID observation for $\xi=42-75^{\circ}$. Parameters for the most southern irregularity are given in lower part of panels 1,2 , and 3; parameters for the rest of irregularities are given in upper part of these panels.

quasi-periods $L$ upon the distance between their midpoints and the source for various distances $R_{1}$.

Figure 15 shows the data accumulated at midnight summer time during minimal and small solar and magnetic activity. In this experiment the MS TIDs were observed in a layer with half-thickness $H=50 \mathrm{~km}$, though such values in our experiments were seldom observed. The TID amplitude for most irregularities registered in this satellite pass was $A_{d}=0.08$. If a TID wave propagated in the north-south direction, the most intense spectral component of the irregularity period was $L=425 \mathrm{~km}$. As in previous experiments we observed that the $\xi$ was negative and the function
$L(\varphi)$ may be approximated by a linear regression $(q=0.63)$. It turned out that functions $L(\varphi)$ are decreasing and TIDs were observed to the south of the measuring station. The above observations indicate that the excitation source of registered TIDs was located at high latitudes.

Table 8 shows the estimated values of distance $R$ between a TID source and the measuring station calculated using formula (5). It was assumed that the energy of the precipitating particles is released at an altitude of $200 \mathrm{~km}$ (see also Fig. 6). As can be seen, the distance from the source was in the range 650$1900 \mathrm{~km}$. 

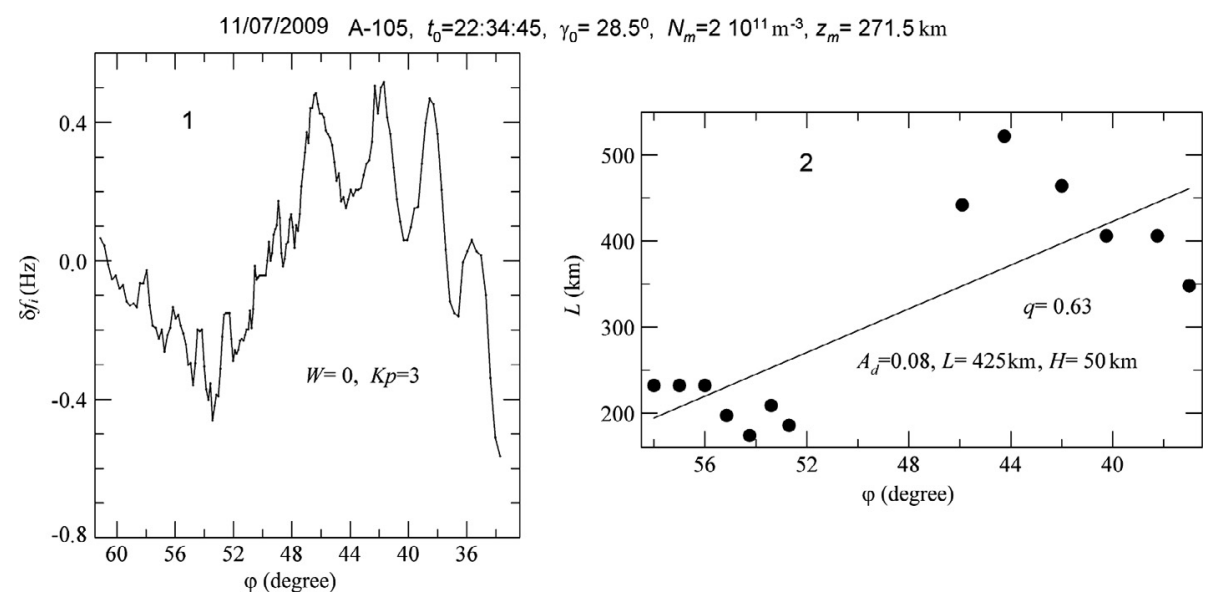

Fig. 15. The results of MS TID observation for $\xi=-(50-62)^{\circ}$.

Table 8. The distances between the MS TID and measuring station, shown in Figures 14 and 15.

\begin{tabular}{lcc}
\hline \hline Date & $\xi$ (degrees) & $R(\mathrm{~km})$ \\
\hline $14 / 01 / 1999$ & $-(43-59)$ & 650 \\
$23 / 07 / 1999$ & -75 & 1900 \\
$09 / 11 / 2004$ & $-(42-59)$ & 600 \\
$11 / 07 / 2009$ & $-(50-62)$ & 750 \\
\hline
\end{tabular}

To verify the above calculations we estimated distances to the disturbance source by another method. As noted above, the TID quasi-periods, defined by our model, are increasing as distance from the disturbance source increases. As mentioned above, the specific distribution of spatial quasi-periods in the TID train is observed for selected values of $R_{1}$ (see Fig. 4). Therefore, if the experimentally observed distribution is approximated by the function $L(R)$ predicted by the model for the chosen $R_{1}$ values, the distances between the disturbance source and each quasi-period can be specified. To illustrate the calculations we choose the three values of quasi-periods shown in Figure 14 (panel 2), since these data have the highest correlation coefficient of linear regression. The approximation for the function $L(R)$ was made using a sampling increment for $R_{1}$ equal to $500 \mathrm{~km}$. The results are illustrated in Figure 16. The model error relative to the experimental data is equal to $5.8 \%$. Figure 14 (panels 1 and 2 ) indicates that the quasi-period $L$ closest to the measuring station (latitude $49.63^{\circ} \mathrm{N}$ ) has the smallest length. As can be seen from Figure 16, the distance to the middle of this quasi-period is $750 \mathrm{~km}$, which is close to the distance between the source and the measuring station, $R=650 \mathrm{~km}$, estimated by $\xi$ values, averaged over the satellite pass (see Table 8).

\subsection{Earthquake in South Sumatra}

Figure 17 shows the data recorded September 30, 2009 and October 01, 2009 around midnight during a period of low magnetic and low solar activity. The local time of observation, the magnetic and solar activities were similar to those in the experiment whose results are shown in Figure 15. However, in contrast to those data and the data illustrated in Figure 14, the linear regression of $L(\varphi)$ presented in Figure 17 is a decreasing function of latitude $\varphi$. The TIDs are observed in the northern part of the $\delta f_{i}(\varphi)$ registration chain. According to our model, this

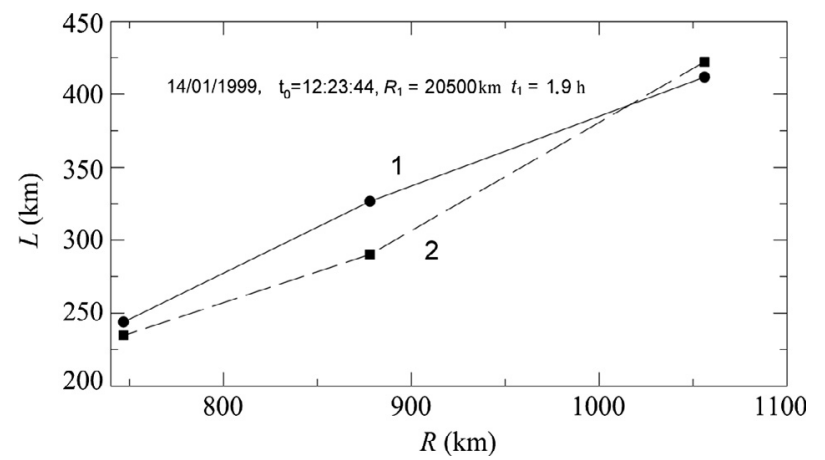

Fig. 16. 1 - the experimental curve $R(L) ; 2$ - the curve $R(L)$, predicted by the model.

suggests that the source of these TIDs is located to the south of the southern boundary of radio visibility $\left(35^{\circ} \mathrm{N}\right)$, and that the disturbance moves from the south to the north.

We found that the same propagation direction was also observed in other registration cycles during magnetic storms. In these rare cases, the location of the magnetospheric cusp was located south of or above the measuring station. These conclusions were made by calculation of the cusp location using empirical models by Köhnlein \& Raitt (1977) and Deminov et al. (1992), which defines the cusp position depending on the longitude and geomagnetic activity. The experimental data presented in Figure 17, correspond to low magnetic activity, therefore the magnetospheric cusp was excluded as AGW source.

We took into account the fact that the above-mentioned data were registered after the earthquake in South Sumatra (Indonesia) at 30/09/2009 13:16:08.6 MST (10:16:08.6 UT). The earthquake magnitude was 7-7.5, the depth was $90 \mathrm{~km}$, the epicenter coordinates were $0.84^{\circ} \mathrm{S}, 99.8^{\circ} \mathrm{E}$. The azimuthal angle to the epicenter measured from north was $\zeta=111.3^{\circ}$ and the distance between the epicenter and the measuring station $R_{\text {quake }}=8200 \mathrm{~km}$.

The registrations of $\delta f_{i}(t)$, obtained after the earthquake and their approximation by the model under unperturbed and perturbed conditions, are presented in Figure 18 on the left and right panels, respectively. Adaptation of the global space-time regular model of $N$ was carried out using the method described by Tyrnov et al. (2005). The values of $N_{m}$ and $z_{m}$ shown in the figure were calculated from model values corrected for the 
30/09/2009, A-103, $t_{0}=22: 36: 48, \gamma_{0}=2.7^{0}, N_{m}=1.210^{11} \mathrm{~m}^{-3}, z_{m}=263.5 \mathrm{~km}$
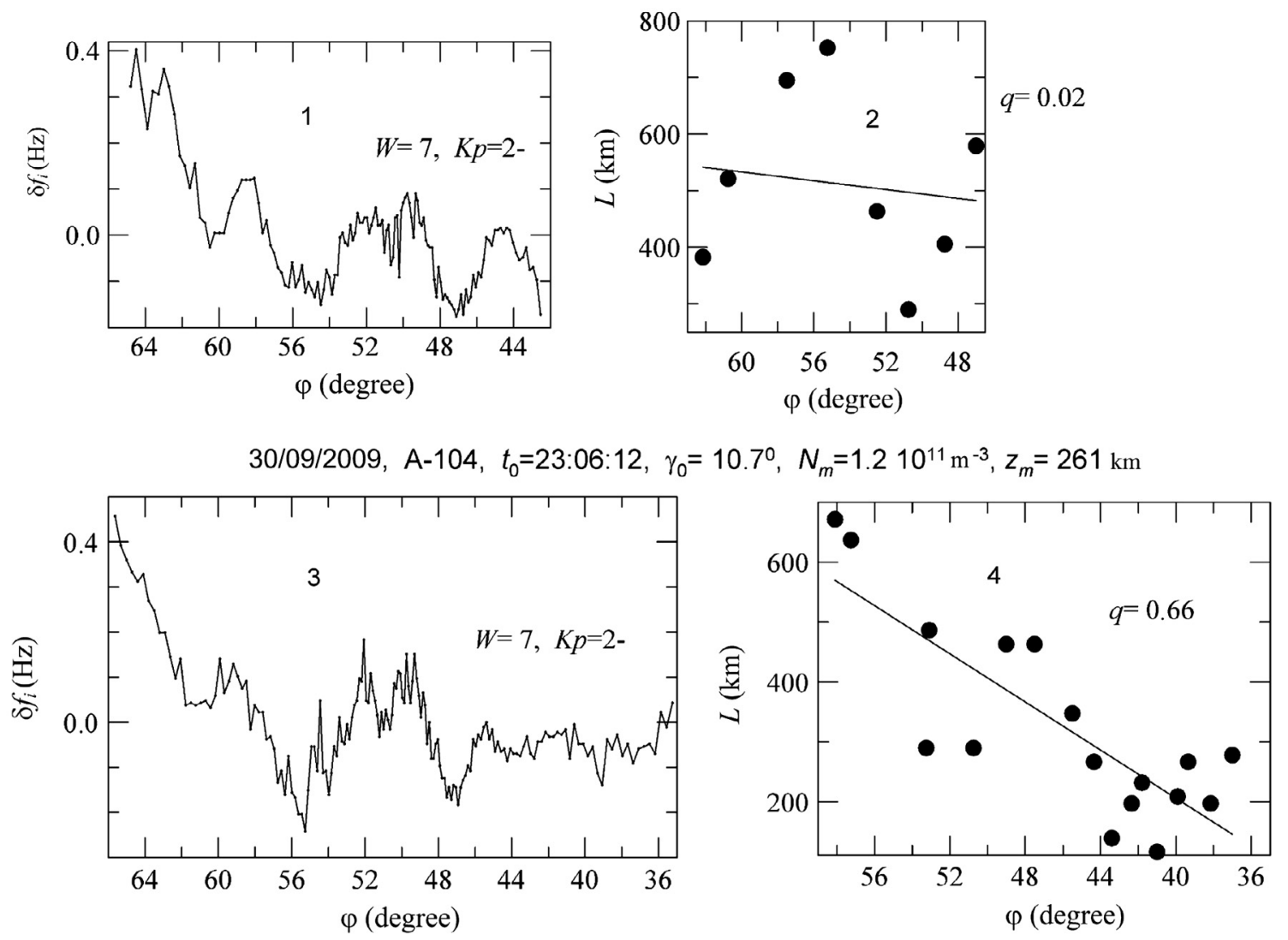

01/10/2009, A-105, $t_{0}=01: 54: 00, \gamma_{0}=3.7^{0}, N_{m}=0.9310^{11} \mathrm{~m}^{-3}, z_{m}=249 \mathrm{~km}$
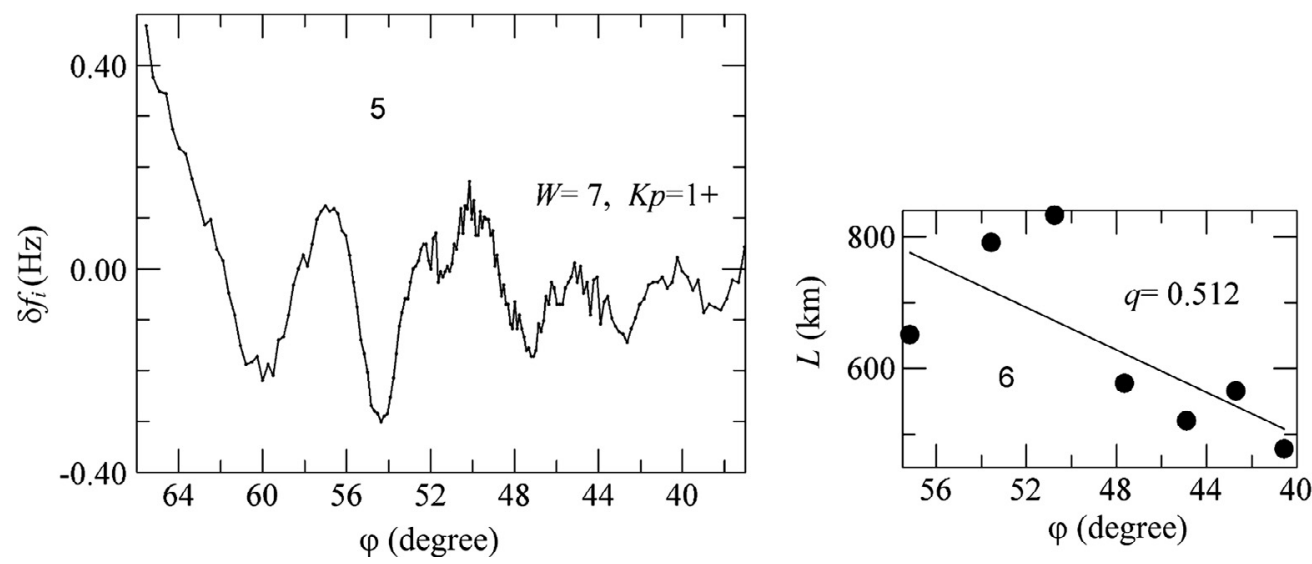

Fig. 17. The plots $\delta f_{i}(\varphi)$ and $L(\varphi)$, registered after South Sumatra Earthquake.

measuring station coordinates. The TID parameters were estimated by an iterative technique, using the possible range of the parameters defined during the direct problem solution. We have not always achieved exact phase matching between the model and experimental curves, since our main objective was to estimate the spatial scale and amplitudes of the disturbances. To avoid cluttering, the tick marks at the time axis origin indicate the hours and minutes and further along the axis only minutes of a current hour. We found that when an MS AGW, formed by extrema numbers 7, 8, and 9 (inferred from the model), propagates from the epicenter to the measuring station, it transforms into an LS AGW and, in addition, the azimuthal angle of the wave vector changes from 111.3 to $\sim 150^{\circ}$. We believe that such effect may be explained by regional winds on the propagation path. A less probable cause may be regional anomalies of pressure and temperature. As can be seen, in all three sounding intervals the MS TID with $L=2134 \mathrm{~km}$ has the largest amplitude $A_{d}=0.6$. All parameters of this TID: $L$, $A_{d}, H, \xi$, and $\Phi$ did not change for at least $3.3 \mathrm{~h}$. In the years close to solar activity maximum, the lifetime of the AWG track in the ionosphere can be more than $5 \mathrm{~h}$. Therefore, the $\delta f_{i}(t)$ registrations shown on panels 2 and 3 indicate that in this experiment the wind velocity component along the AGW wave vector was negligible.

The $\xi$ value for the TID of any scale is $80^{\circ}$. The positive sign and large value of $\xi$ indicate that the disturbance source is located thousands of kilometers to the south of the measuring station. Above, we have identified the source that gave rise to 


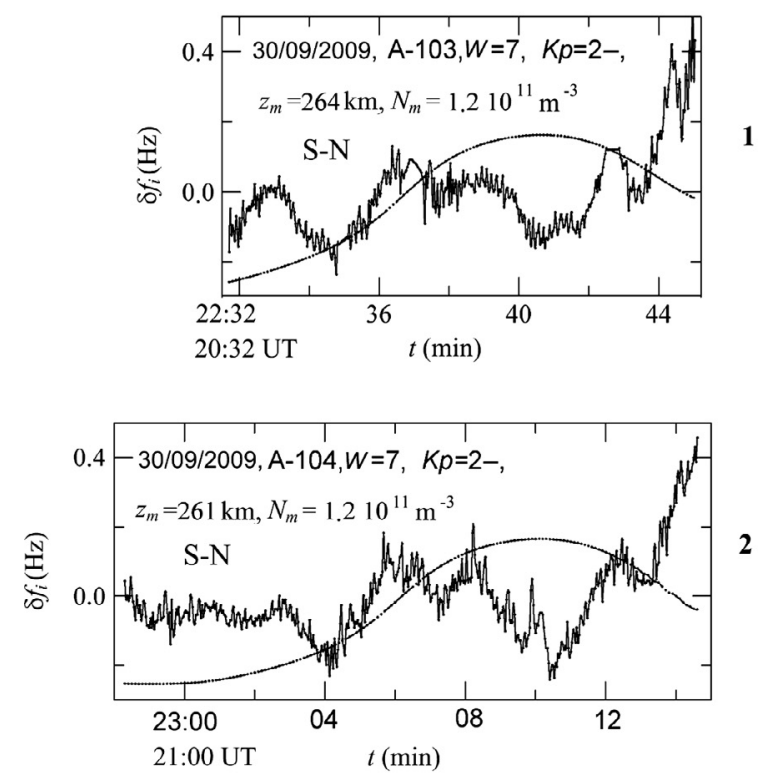

$L_{1}=2134 \mathrm{~km}, A_{d}=0.7, \xi=80^{\circ}, H=80 \mathrm{~km}, \Phi=70^{\circ}$
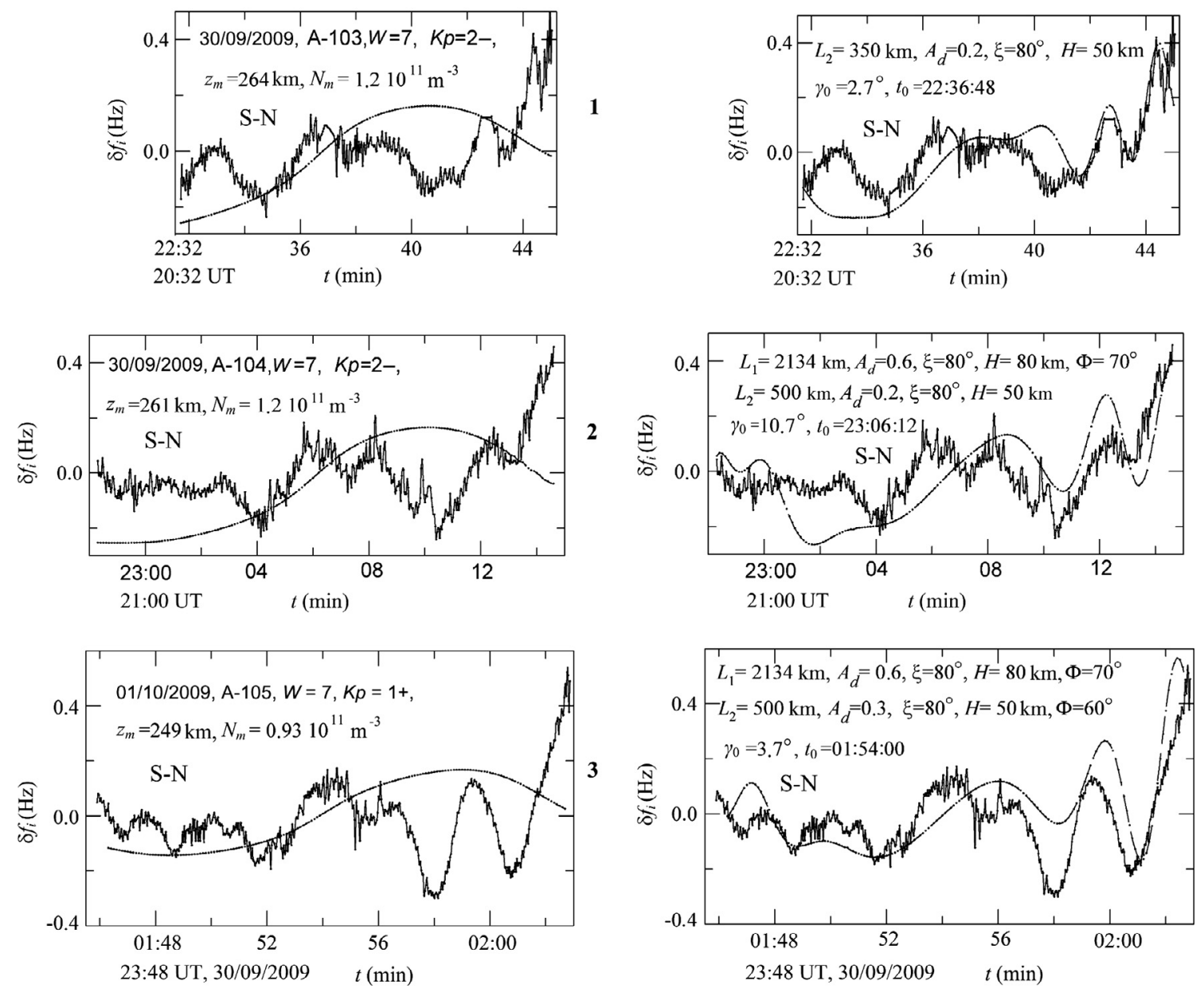

Fig. 18. The recordings of $\delta f(t)$ obtained after South Sumatra Earthquake (solid lines) and their model approximations for undisturbed conditions (left panel) and for disturbed conditions (right panel). "S-N" - satellite moves from the south to the north; "N-S" - satellite moves from the north to the south.

the LS TID and now we consider an MS TID with $L=350 \mathrm{~km}$ and $A_{d}=0.2$ (see panel 1). The amplitude of this irregularity stayed constant for about $30 \mathrm{~min}$ while the period $L$ was increasing to $500 \mathrm{~km}$ (panel 2). Then the period did not change within $2.8 \mathrm{~h}$, but the amplitude increased to $A_{d}=0.3$. Undoubtedly, these MS TIDs are also generated by a southern source, but in our opinion, they cannot be related to the earthquake because low-speed extrema, observed as an MS TID, should be observed later, but not simultaneously with an LS TID.

A series of earthquakes with magnitude from 6 to 7.5 occurred prior to the above-mentioned earthquake and the shocks nearest in time were observed at 8:25:46 and 11:24:57 MST (05:25:46 and 8:24:57 UT), respectively. The first shock took place in Tonga Islands $\left(15.56^{\circ} \mathrm{S}, 173.49^{\circ} \mathrm{W}\right.$, $\left.R_{\text {quake }}=15368 \mathrm{~km}, \zeta=134\right)$, and the second in Samoa Island $\left(16.72^{\circ} \mathrm{S}, 172.87^{\circ} \mathrm{W}, R_{\text {quake }}=15513 \mathrm{~km}, \zeta=134^{\circ}\right)$ at a depth of $10 \mathrm{~km}$. These shocks occurred 2 and $5 \mathrm{~h}$ ahead of the AGW, generated by the South Sumatra earthquake, and their epicenter azimuths are approximately equal to that of the AGW source. A superposition of the extrema, generated by these earthquakes, could give rise to the MS TID in the sounding region. According to our estimates, the extrema of each of these earthquakes at delays equal to $\sim 11.5$ and $14.5 \mathrm{~h}$ could generate over the measuring station only LS TID $(L=1500 \mathrm{~km})$. The distance between the earthquake epicenters was equal to $\sim 15000 \mathrm{~km}$, and it does not contradict this hypothesis, since disturbances, arising from high-altitude nuclear explosions with an energy in the range from 0.02 to 1.4 Kt TNT, were observed at similar distances (see Sect. 4).

\subsection{The time dynamics of $L$ and $A_{d}$}

According to our TID model, initial values of velocity extrema $V_{i}$ differ very much. Thus, even adjacent initial spatial half-periods in a TID train differ essentially in magnitude. This effect has always been observed experimentally in the first hours after the initial disturbance. Registrations of $\delta f_{i}(t)$ of MS TID are illustrated in Figure 19 where all identified disturbances were moving from the north to south, i.e., from the cusp to the equator. The direction of TID movement is unambiguously defined by the $\xi$ sign. The excitation time of the TIDs, detectable in registrations presented in panels 1 and 2, was about 19:00 and 21:00, respectively. Our estimates show that location and energy of the TID sources were almost identical in both events, considering the very similar manifestation of disturbances (see panels 1 and 2) and on almost equal delays relative to the excitation time (about $2 \mathrm{~h}$ ). The TID excitation time inferred from the registrations $\delta f_{i}(t)$ is a characteristic indicator for high-energy proton precipitation into the magnetospheric cusp. 
J. Space Weather Space Clim. 3 (2013) A30
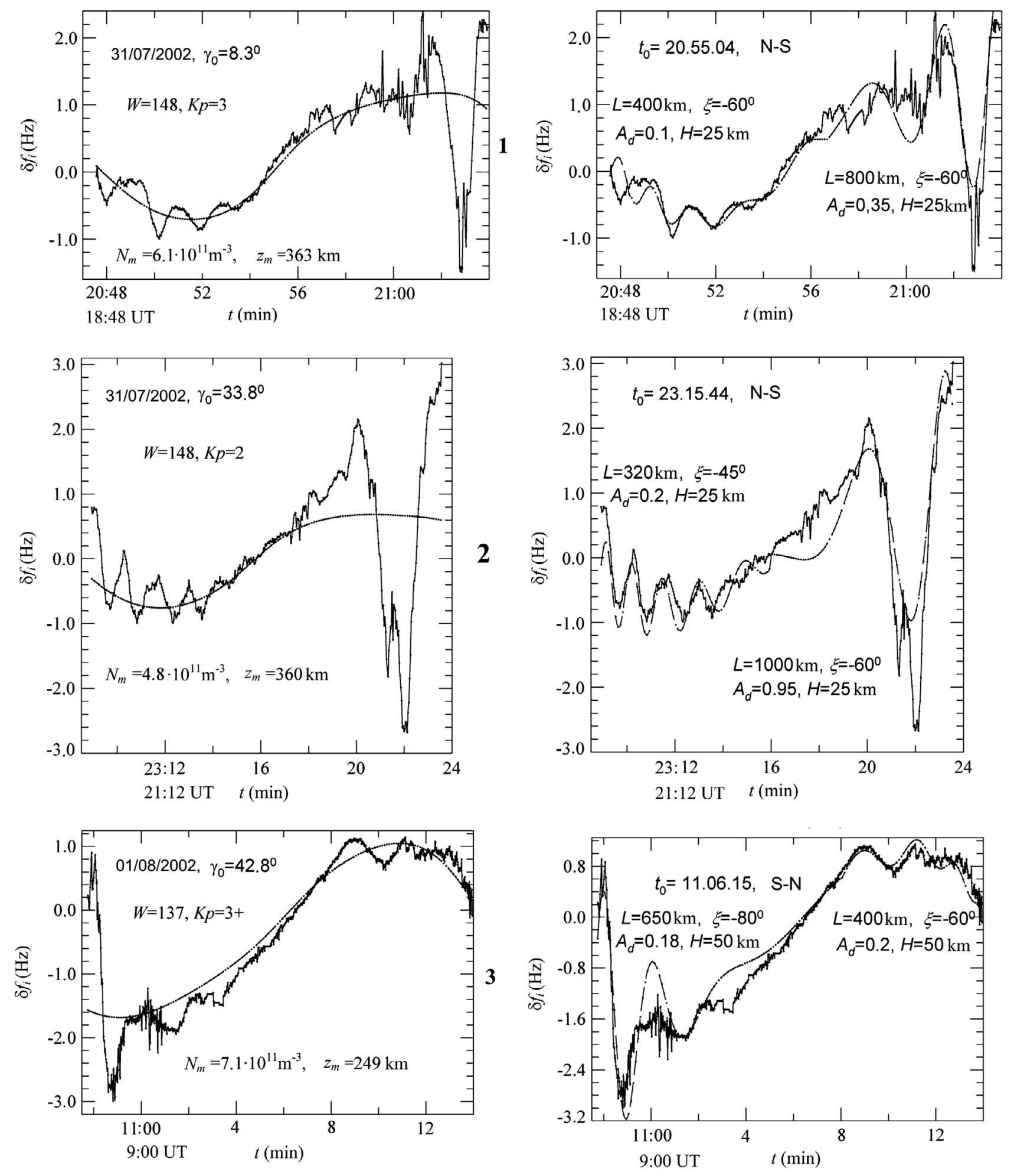

Fig. 19. Examples of MS TID with $A_{d}=0.18 \ldots 0.95$ and $\xi=-45 \ldots-80^{\circ}$. The recordings of $\delta f(t)$ obtained after precipitation energetic protons near the magnetospheric cusp (solid lines) and their model approximations for undisturbed conditions (left panel) and for disturbed conditions (right panel). "S-N" - satellite moves from the south to the north; "N-S" - satellite moves from the north to the south.

The dependencies noted above show that in the first hours after the AGW initiation $L$ and $A_{d}$ are substantially increasing with increasing distance from the excitation source. Tsugawa et al. (2007a) observed the effect of TID amplitude increase but did not explain it. However, the effect is predicted by our model. The effect was also observed in our study. Thus, the data presented in the first and second panels show that the periods are increasing from 400 to $800 \mathrm{~km}$ and from 320 to $1000 \mathrm{~km}$, respectively (increase from 2 to 3 times), during the second hour after excitation. The observations recorded $14 \mathrm{~h}$ after TID excitation and presented in the third panel show the period increasing from 400 to $650 \mathrm{~km}$. This suggests that these disturbances are generated by subsequent slower quasiperiods of the AGW train for which, according to the model, 

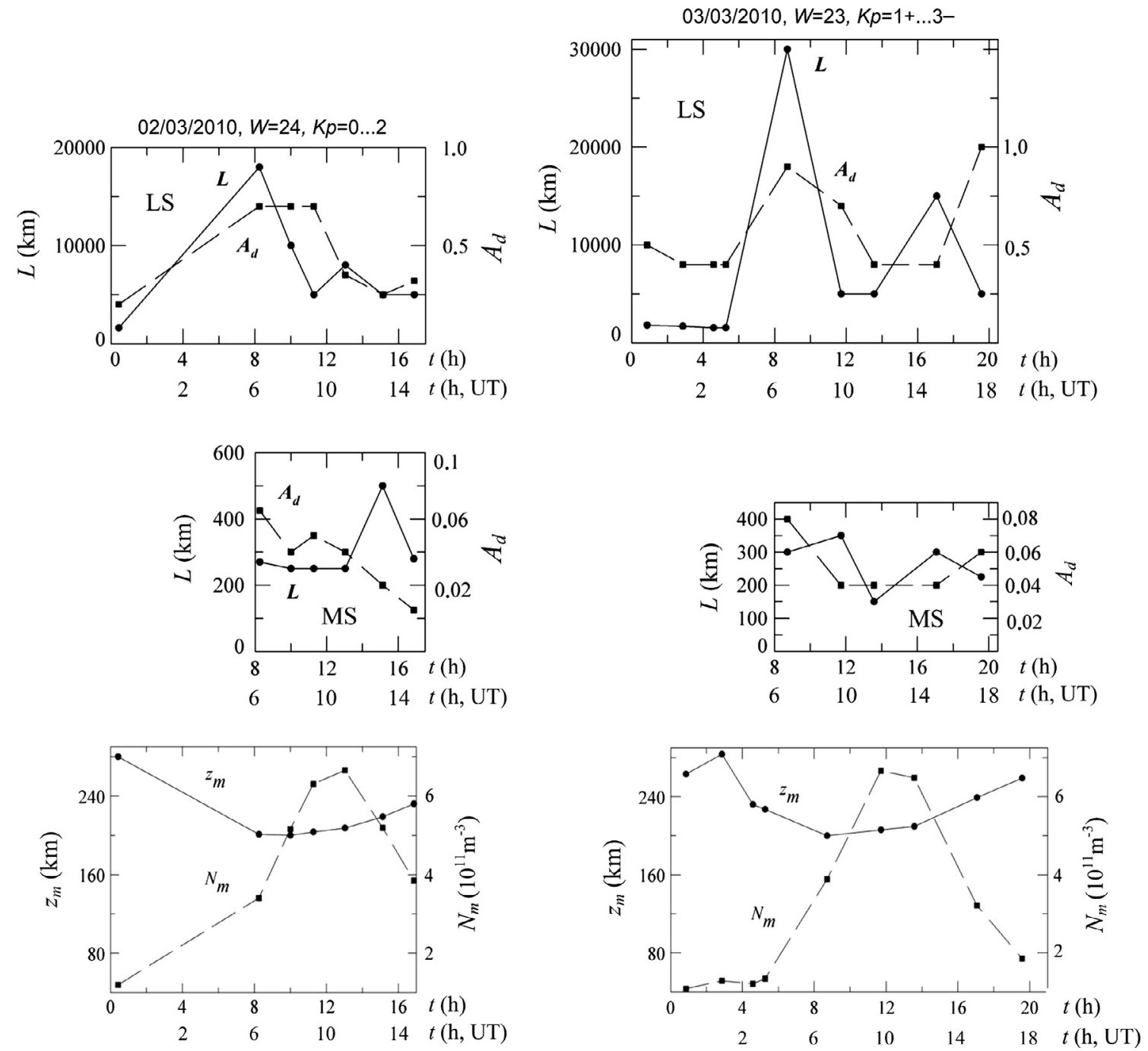

Fig. 20a. The diurnal dependencies of $L, A_{d}, z_{m}$, and $N_{m}$, registered on 2 and 3 of March 2010.

the period increases much less. Increasing of $L$ and $A_{d}$ for MS TID moving to the equator over a region with a typical extent of about $3000 \mathrm{~km}$ gives further evidence that the source of this TID is at northern latitudes. Using a large data set and another methodology, Fedorenko et al. (2010a) have ascertained that the $L$ values are increasing if the latitude is decreasing.

Using $\delta f_{i}(t)$ registrations, we found that when there exist only the MS TIDs or MS TIDs and LS TID simultaneously, a general rule can be formulated as: "the larger is $L$, the greater is the relative amplitude $A_{d}$ ". For LS and MS disturbances the ratio between $A_{d}$ values may be up to 10 . This rule is predicted by our model and is confirmed in most of our experiments. A similar correlation has been established by Fedorenko et al. (2010a) using statistical data analysis for MS TID.

Additional confirmations of our findings are shown in Figures 20a and 20b, where for low solar activity ( $W=18-25$ ) the diurnal variations of $L, A_{d}, N_{m}$, and $z_{m}$ above the measuring station for the period between $02 / 03 / 10$ and $05 / 03 / 10$ are shown. The diurnal data $L(t)$ and $A_{d}(t)$ were obtained by $\delta f_{i}(t)$ registrations using the method proposed by Fedorenko et al. (2012), which allows to identify the TID having a spatial scale in the range $200-35000 \mathrm{~km}$ and relative amplitudes from $\sim 0.02$ to 1 over an observation region with a radius of about
$1500 \mathrm{~km}$. This method may also be used to analyze MS TIDs and LS TID simultaneously observed over the same region.

The plots $L(t)$ and $A_{d}(t)$ for LS TID and MS TID are presented in Figures 20a and 20b, respectively. If LS and MS TIDs were observed in the southern and northern parts of the registration station chain and have different $L$ values, then their average values are indicated in the figure. We have estimated that for the LS TID with $L \approx 1500-35000 \mathrm{~km}$ the amplitudes $A_{d}$ are in a range $0.4-1$, and for MS TID with $L \approx 150-800 \mathrm{~km}$ in the range $A_{d} \approx 0.02-0.5$. We believe that the LS TIDs, presented in Figures 20a and 20b, are generated near midnight.

The saw-tooth curves of $L(t)$ may be explained by large differences in adjacent spatial periods at the beginning of the TID train. Such dynamics are readily explained by our semi-empirical model.

An important feature of the data obtained during minimum solar activity is that when a disturbance starts, an LS TID is generated, and a significant decrease in $N$, as compared to the unperturbed background values, is observed. Such volume of reduced density is known as a hole. According to our model, oscillations in a disturbance source start with lowering air pressure up to two times (see Table 1). 

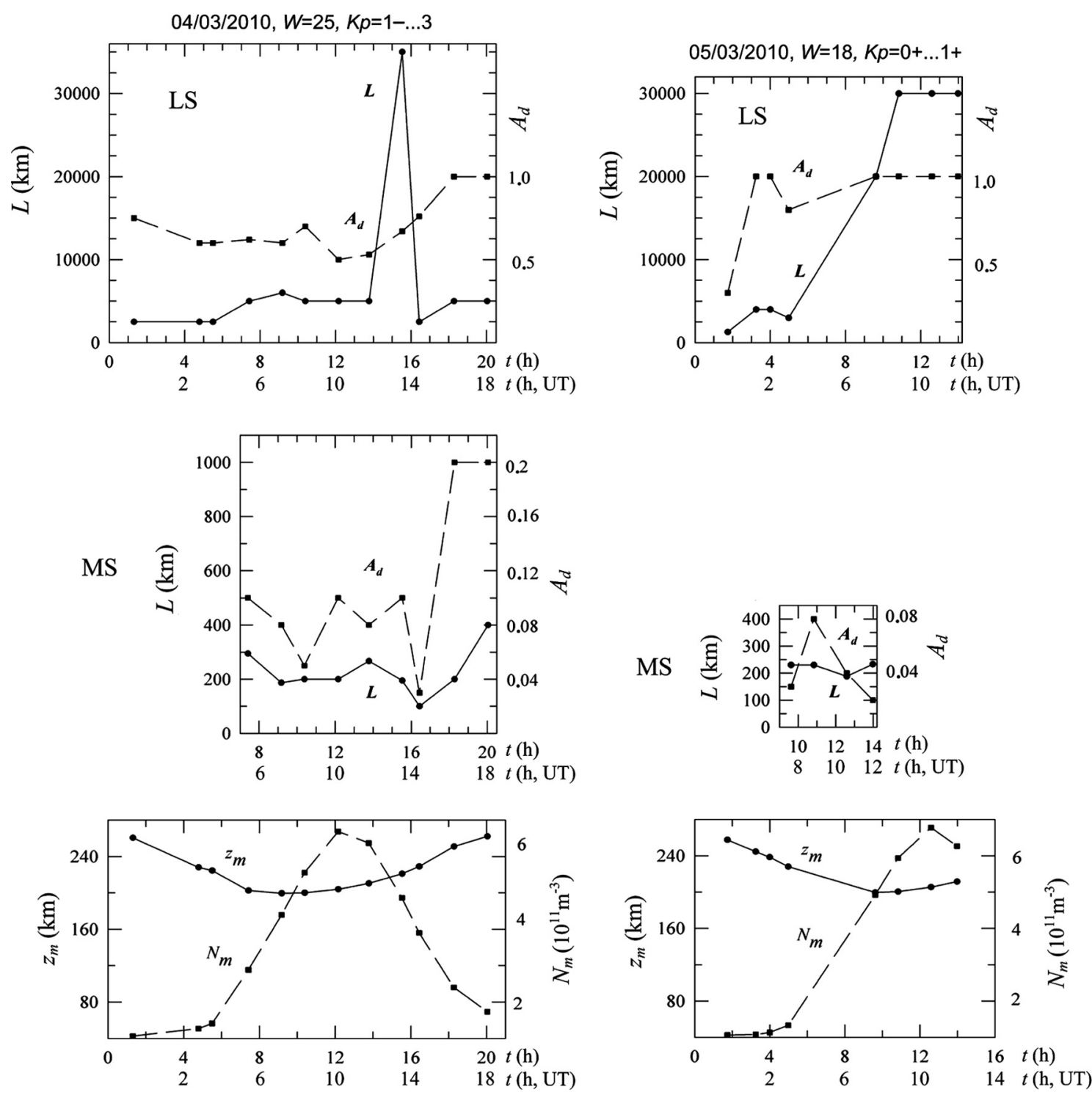

Fig. 20b. The diurnal dependencies of $L, A_{d}, z_{m}$ and $N_{m}$, registered on 4 and 5 of March 2010.

This area of reduced air pressure propagates as a wave in the horizontal plane in all directions at $11 \mathrm{~km} \mathrm{~s}^{-1}$. In our experiment, an area of reduced atmospheric pressure was identified by monitoring $N$. The latitude of minimum values of $N$ within such volume is moving in the range from $\sim 60^{\circ}$ to $40^{\circ} \mathrm{N}$. A relative amplitude $A_{\text {low }}$ of holes in this latitude range is reduced between 1 and $\sim 0.3$ ( $A_{\text {low }}=\Delta N_{\text {low }} / N_{n}$, where $\Delta N_{\text {low }}$ is the absolute decrease of $N_{m}$ in a hole and $N_{n}$ is the unperturbed $N_{m}$ ). These changes were recorded during the daily observation periods (lasting for about 15-20 h). Thus, during the day (in fact, from one event of particle participation to another) we observed a significant decrease in the background $N$ levels during the minimum of solar activity ( $W=18-25)$. As noted in Section 4 the significant decrease of $N$ was also observed after nuclear experiments in 1962, at low solar activity $(W=11-28)$.

According to our observations, TIDs are generated frequently at high latitudes (in the magnetospheric cusp area) and can be observed in the midnight sector. The TIDs may be excited in the morning cusp and traverse the observation point after passing over the North Pole. Here we present only the observation results without analysis of the process in detail. Often the event (precipitation of energetic protons) occurs once every 2 or 3 days, but sometimes it may appear two or three times per day (see, Fig. 19). In the latter case, $L$ and $A_{d}$ have no time to decrease to values normally observed at the end of the day with a single disturbance source (see, for example, Fig. 20a for 02/03/2010). Rather, a spatial AGW trace existing in the ionosphere may be superseded by a new trail having larger amplitudes and quasi-periods. If the time between consecutive precipitation events is more than a day, the values of $L$ and $A_{d}$ are reduced to their minima reaching $\sim 150 \mathrm{~km}$ for $L$ and up to several percent for $A_{d}$.

From the data shown in Figure 20, we may conclude that the values of $L$ and $A_{d}$ decay very slowly over about $5 \mathrm{~h}$. This effect may be explained by our model, which states that only the first two periods of an AGW train have maximum $A_{d}$ and subsequent periods are decreasing linearly with time. Due to differences between adjacent train velocities $V_{i}$, decreasing from one to another half-period, the corresponding values of $L$ are also reduced, at first very sharply and then only little. Consequently, the third, fourth, and subsequent half-periods of an AGW train could not erase the previous long-living AGW trace in the ionosphere and replace it by lower values of $L$ and $A_{d}$. Therefore, this large-scale and large amplitude trace stays in 

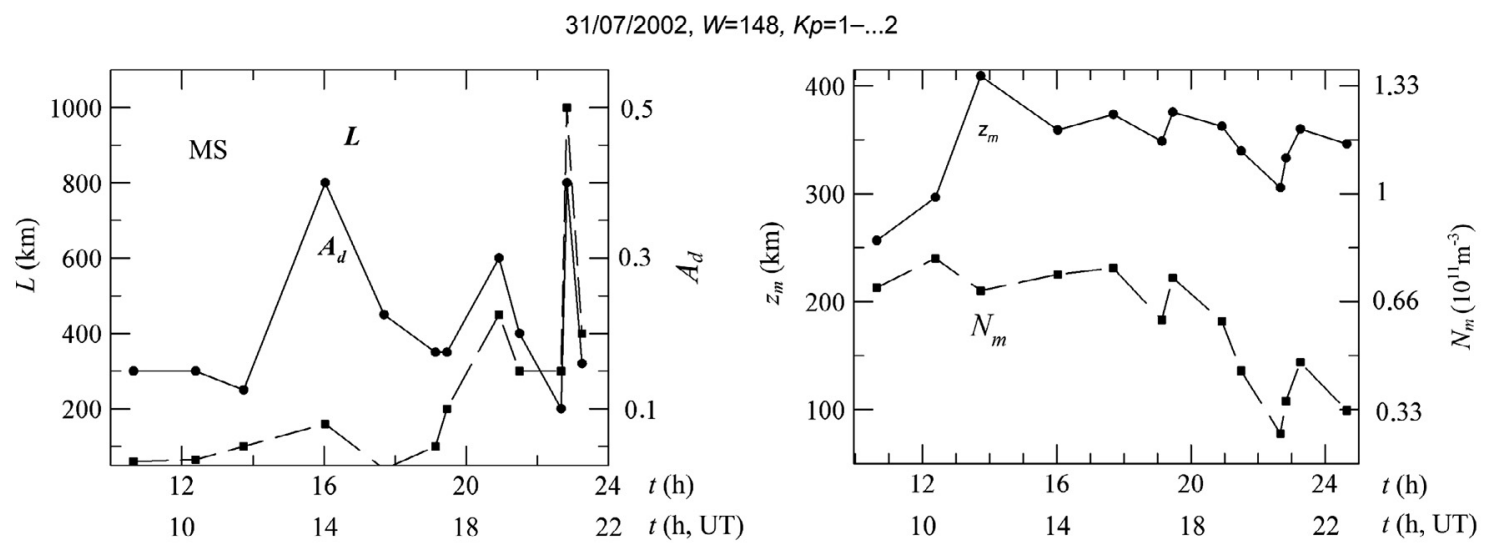

$01 / 08 / 2002, W=137, K p=1 \ldots 5+$
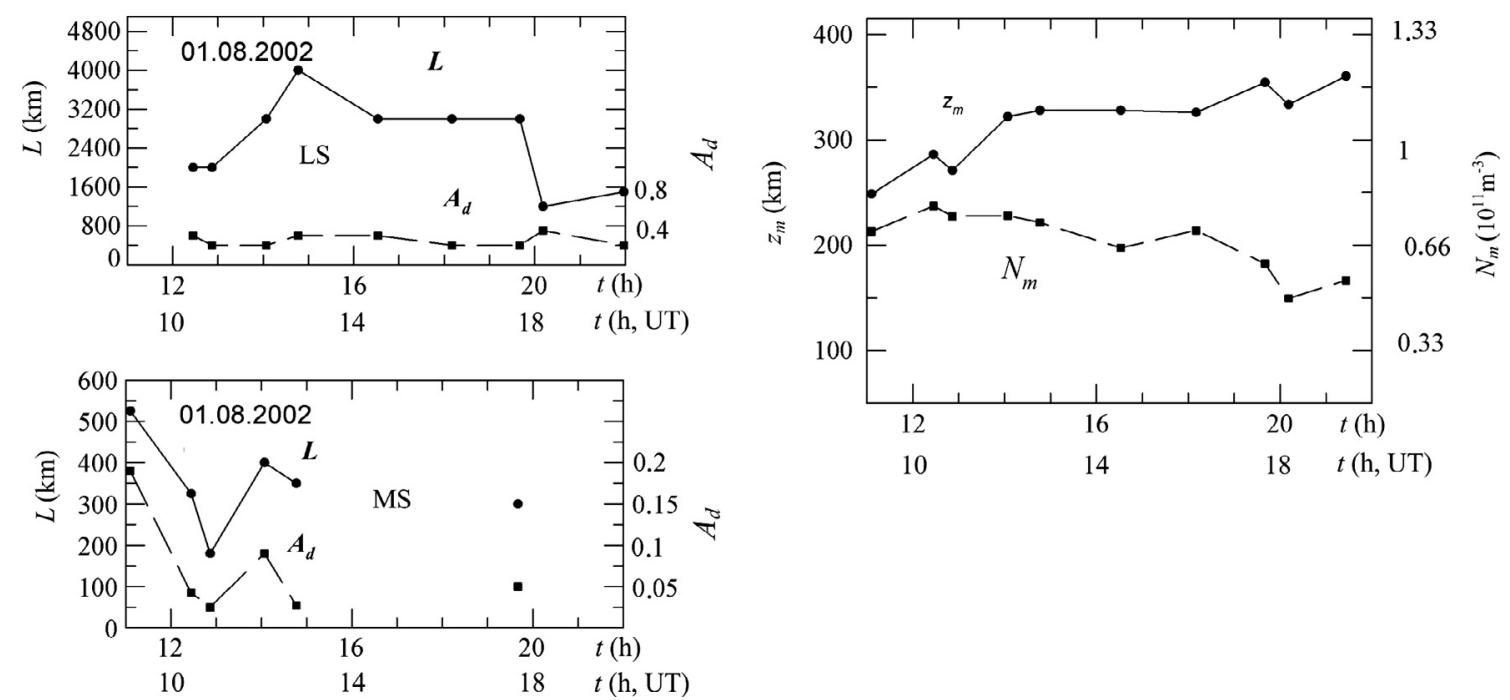

Fig. 21. Dynamics of parameters for TIDs and F2 maximum, observed on 31 July and 1 August 2010.

the ionosphere without being influenced by subsequent TID phases. Only quasi-periods of a train following much later can establish a new TID with lower values of $L$ and $A_{d}$.

During daytime and, particularly around noon when background electron density is increased as compared to night-time values, the MS AGW train can be observed to be superposed over an existing long-living LS TID. If it propagates with significantly lower values of $L$ and $A_{d}$, the original LS TID track becomes modulated by the spatial quasi-harmonics of the MS TID.

Figure 21 is similar to Figure 20, but the data were collected during maximum solar activity (from 31/07/2002 $(W=148)$ to $01 / 08 / 2002(W=137))$. Sharp increases of $L$ on $31 / 0 / 2002$ at $\sim 19: 00$ and 22:40, and on 01/08/2002 at 12:27 are attributed to separate precipitation events of energetic protons. Saw-tooth pieces are present in the $L(t)$ curves registered both for the minimum and maximum solar activity, but these effects are manifested in different ways.

The maximum values of periods $L_{\max }$ reach 30000 $35000 \mathrm{~km}$ and about $3200-4000 \mathrm{~km}$ during daily observations in periods of solar minimum and maximum activity, respectively. The nearly tenfold reduction of the maximum values of $L_{\max }$, for solar maximum, is explained by a significant decrease of the disturbance lifetime, since the highest-speed LS TIDs could not be recorded because of the limited cadence of our measurements which was equal to $\sim 1.5-2 \mathrm{~h}$. Such a large difference of $L_{\max }$ for minimum and maximum solar activity can be partially explained by the different delays of the first extremum, which is $1-2 \mathrm{~h}$ and $11 \mathrm{~h}$ for minimum and maximum solar activity, respectively.

We found that regions with reduced electron density were practically never observed under conditions of maximum solar activity, but areas with high density relative to unperturbed background values were detected during the whole measurement period. On 01/08/02 the disturbance amplitude $A_{\text {high }}$ in this region changed in the range $0.3-0.6$ for about $20 \mathrm{~h}$ according to the quasi-harmonic law. Here $A_{\text {high }}=\Delta N_{\text {high }} / N_{n}$; $\Delta N_{\text {high }}=\left|N-N_{n}\right| ; N$ and $N_{n}$ are disturbed and undisturbed electron concentrations, respectively. The latitude of the center of this region fluctuated in antiphase with $A_{\text {high }}$ around $\sim 45^{\circ} \mathrm{N}$. The amplitude of these fluctuations was in the range $3^{\circ}-5^{\circ}$, and the period was increasing almost linearly from 100 to $200 \mathrm{~min}$ during the time interval $\sim 13: 15-16: 30$. Only one LS TID was recorded on $31 / 07 / 02$ at $\sim 19: 30$. The TID parameters were found to be $L=5000 \mathrm{~km}, A_{d}=0.9$, and $A_{\text {high }}=2.5$.

\subsection{Spatial dependence of TID inclination angle $\xi$}

From the analysis in Section 2 we deduced that the further a TID quasi-period is away from the excitation source, the greater 
Table 9. The relation between $\xi$ and $L$ for the TID presented in Figure 19.

\begin{tabular}{lcrc}
\hline \hline Panel \# & $\begin{array}{c}\text { The part of } \delta f_{i}(t) \\
\text { registration }\end{array}$ & $L(\mathrm{~km})$ & $\xi\left(^{\circ}\right)$ \\
\hline 2 & North & 320 & -45 \\
3 & South & 1000 & -60 \\
& North & 400 & -60 \\
& South & 650 & -80 \\
\hline
\end{tabular}

is the absolute value of $\xi$. The effect is clearly visible in Figure 19 (panels 2 and 3) and is summarized in Table 9. The parameters: $L, A_{d}$, and the absolute value of $\xi$ increase while the TID is moving away from the excitation source. The front of an MS TID is tilted in the direction of disturbance propagation.

This tendency also holds for LS TIDs. However, if $L>900$ $1000 \mathrm{~km}, \xi$ can be determined with an error not less than $\pm 30^{\circ}$. In many cases, one can only determine a sign of $\xi$ and conclude that $\xi$ is within an expected range (Fedorenko et al. 2012). See, for example, five cases in the article Fedorenko et al. (2012) for $L=1800-2500 \mathrm{~km}$ and $A_{d}=0.5-0.75$, obtained at night under solar minimum conditions. For $L>2000-3000 \mathrm{~km}$ the absolute value of this angle, in some cases, can be determined only with accuracy $\delta \xi \sim 50-70^{\circ}$. These restrictions are imposed by the geometry of radio sounding for large $L$.

\section{Summary}

In this article we have discussed the following observations and their implications:

1. Large-and medium-scale TIDs are different stages of an initial perturbation generated by the same source. The spatial and temporal dynamics of the TID parameters may be predicted by our semi-empirical model. The model was verified via analysis of observations published in the scientific literature. Our results show that the spatial and temporal periods are predicted with accuracy not greater than $12 \%$.

2. The following parameters of TIDs: the spatial period, amplitude, and front inclination angle in a vertical plain increase with decreasing latitude in almost all observations made during the two solar cycles in the range of latitudes $\sim 33-66^{\circ} \mathrm{N}$. This indicates that the sources of most TIDs of natural origin are located at high latitudes.

3. Spatio-temporal dynamics of the TIDs, generated presumably by the precipitation of energetic protons in the magnetospheric cusp of the northern hemisphere, are described by our empirical model.

4. The semi-empirical model predicts the saw-tooth pieces on time dynamics of spatial TID periods observed in our transionospheric radiosounding experiments.

5. Directly proportional relationship between the amplitude and horizontal spatial period of TIDs was observed during the minimum and maximum of solar activity.

6. No systematic diurnal and seasonal variability of TID occurrence was found, but the TID occurrence was growing from $\sim 50 \%$ in 1987 to $\sim 98 \%$ in 2010 . The results of other studies suggesting that TID occurrence does not depend on sunspot number and magnetic activity are confirmed.
7. For the period 1987-2010 the occurrence of TIDs, propagating in the free-wave mode and waveguide mode, is $76 \%$ and $24 \%$, respectively.

8. The minimum values of the horizontal scale of TIDs do not exceed $\sim 150-200 \mathrm{~km}$, predicted by our model and confirmed by observations.

Acknowledgments. We are grateful to all researchers for their contribution to theoretical and experimental studies of TID since their first observation by Munro (1948). We also express our special sincere gratitude to participants of nuclear experiments for their devotion, and to scientists, who have used these events for geophysical researches and have published their results, frequently with frank remarks and doubts. Their contribution to development of our model is invaluable. We are grateful to the editor and two anonymous reviewers for useful recommendations and questions.

\section{References}

Adushkin, V.V., and K.I. Gorelyi, The response of the ionospheric Fregion to atmospheric nuclear explosions, Dynamic Processes in Geospheres, Geophysics of Strong Disturbances. Collected Scientific Transactions (in Russian), Moscow, 239-248, 1994.

Afraimovich, E.L., S.V. Voyenkov, K.G. Ratovsky, and P.V. Tatarinov, GPS-detection of the single internal gravitational waves generated during powerful magnetic storms. In: Trudy 21 vserossiiskoi nauchnoi konferentsi po rasprostraneniyu radiovoln (Proc. 21th All-Russia Sci. Conference on Radiowave Propagation), Ioshkar Ola, 1, 124-128, 2005.

Albee, P.R., and D.P. Kanellakos, A spatial model of the F-region ionospheric traveling disturbance following a low-altitude nuclear explosion, J. Geophys. Res., 73 (3), 1039-1053, 1968.

Breitling, W.J., and R.A. Kupferman, Traveling ionospheric disturbances associated with nuclear detonations, J. Geophys. Res., 72 (1), 307-315, 1967.

Bristow, W.A., R.A. Greenwald, and J.C. Samson, Identification of high-latitude acoustic gravity wave sources using the Goose Bay HF radar, J. Geophys. Res., 99 (A1), 319-331, 1994.

Bullough, R.K., and P.J. Caudrey, Solitons, Springer-Verlag, Berlin, Heidelberg, New York, 1980.

Cole, J.D., and C. Greifinger, Acoustic-gravity waves from an energy source at the ground in the isothermal atmosphere, $J$. Geophys. Res., 74 (14), 3693-3703, 1969.

Deminov, M.G., A.T. Karpachev, V.V. Afonin, and J. Shmilauer, Change of a position of the main ionospheric trough depending on a longitude and geomagnetic activity, Geomagn. Aeron., 32 (5), 185-188, 1992.

Dorohov, V.L., V.G. Somov, V.N. Fedorenko, and Yu.P. Fedorenko, Diagnostics of medium scale traveling ionospheric disturbances using radio sounding of the ionosphere by signals from loworbiting navigation satellites, Visn. KharkivKarazin Nat. Univ., Ser.: Radiofiz. Elektron. (in Russian), 2 (646), 224-229, 2004.

Evans, J.V., J.M. Holt, and R.H. Wand, A differential doppler study of traveling ionospheric disturbances from Millstone Hill, Radio Sci., 18 (3), 435-451, 1983.

Fedorenko, V.N., Yu.P. Fedorenko, and I.I. Shagimuratov, Results of the ionosphere study by means of diversity reception of radio signals of low-orbiting navigation satellites, Geomagn. Aeron., 37 (3), 346-349, 1997

Fedorenko, Yu.P., O.F. Tyrnov, and V.N. Fedorenko, Parametres of empirical model of traveling ionospheric disturbances, Elektromagnitnye volny i elektronnye sistemy, 13, 21-46, 2008.

Fedorenko, Yu.P., O.F. Tyrnov, and V.N. Fedorenko, Parameters of traveling ionospheric disturbances estimated from satellite beacon observations in low Earth orbit, Geomagn. Aeron., 50, 489-503, 2010a.

Fedorenko, Yu.P., V.N. Fedorenko, and V.L. Dorohov, Front inclination in a vertical plane of Medium-Scale Traveling 
Ionospheric Disturbances and an effective thickness of a layer of their detection, Visn. Kharkiv Karazin Nat. Univ., Ser.: Radiofiz. Elektron., (in Russian), 17 (927), 109-120, 2010b.

Fedorenko, Yu.P., V.N. Fedorenko, and V.N. Lysenko, Parameters of the medium-scale traveling ionospheric disturbances model deduced from measurements, Geomagn. Aeron., 51 (1), 88-104, 2011.

Fedorenko, Yu.P., V.N. Fedorenko, and V.L. Dorohov, Diagnostics of parametres of large-scale traveling ionospheric disturbances with the help radioscopy ionosphere by signals low-orbital navigating satellites, Visn. Kharkiv Karazin Nat. Univ., Ser.: Radiofiz. Elektron., (in Russian), 20 (1010), 97-112, 2012.

Francis, S.H., A theory of medium-scale traveling ionospheric disturbances, J. Geophys. Res., 79 (34), 5245-5260, 1974.

Francis, S.H., Global propagation of atmospheric gravity waves: A review, J. Atmos. Terr. Phys., 37 (6-7), 1011-1054, 1975.

Gossard, E.E., and W.H. Hooke, Waves in the Atmosphere, Elsevier, Amsterdam, Netherlands, 456 p., 1975.

Halperin, Yu.I., L.D. Sivtseva, V.M. Filippov, and V.L. Khalipov, The Sub-Auroral Upper Ionosphere, Nauka, Novosybyrsk (in Russian), 191, 1990.

Heisler, L.H., Anomalies in ionosonde records due to traveling ionospheric disturbances, Aust. J. Phis., 11, 79-90, 1958.

Hocke, K., and K. Schlegel, A review of atmospheric gravity waves and traveling ionospheric disturbances: 1982-1995, Ann. Geophys., 14, 917-940, 1996.

Igarashi, K., S. Kainuma, I. Nishimuta, S. Okamoto, H. Kuroiwa, T. Tanaka, and T. Ogawa, Ionospheric and atmospheric disturbances around Japan caused by the eruption of Mount Pinatubo on 15 June 1991, J. Atmos. Terr. Phys., 56 (9), 1227-1234, 1994.

Iyer, K.N., Effect of traveling ionospheric disturbances on HF phasepath measurements, Indian J. Radio Space Phys., 12, 47-49, 1983.

Kanellakos, D.P., Response of the ionosphere to the passage of acoustic-gravity waves generated be low-altitude nuclear explosions, J. Geophys. Res., 72 (17), 4559-4576, 1967.

Karpachev, A.T., N. Beloff, T.D. Carozzi, P.F. Denisenko, T.J.T. Karhunem, and M. Lester, Detection of large scale TIDs associated with the dayside cusp using SuperDARN Data, $J$. Atmos. Sol. Terr. Phys., 72 (9-10), 653-661, 2010.

Köhnlein, W., and W.J. Raitt, Position of the mid-latitude trough in the topside ionosphere as deduced from ESPO 4 observations, Planet. Space Sci., 25, 600-602, 1977.

Kotake, N., Y. Otsuka, T. Ogawa, T. Tsugawa, and A. Saito, Statistical study of medium-scale traveling ionospheric disturbances observed with the GPS networks in Southern California, Earth Planets Space, 59, 95-102, 2007.

Kunitsyn, W.E., S.N. Suraev, and R.R. Akhmedov, Modeling propagation of acoustic gravity waves in the atmosphere for different surface sources, Vestn. Mosk. Univ., Sert. 3. Fiz. Astron., 2, 59-63, 2007.

Liu, C.H., and K.C. Yeh, Exciting of acoustic-gravity waves in an isothermal atmosphere, Tellus, 23, 150-163, 1971.

Lomax, J.B., and D.L. Nielson, Observation of acoustic-gravity wave effects showing geomagnetic field dependence, J. Atmos. Terr. Phys., 30, 1033-1050, 1968.

Lonngren, K., and A. Scott, Soliton in Actions, Academic Press, New York, San Francisco-London, 1978.

Munro, G.H., Short-period changes in the $F$ region of the ionosphere, Nature, 162, 886-887, 1948.

Obayashi, T., Widespread ionospheric disturbances due to nuclear explosions during October 1961, Nature, 196 (4849), 24-27, 1962

Ogawa, T., K. Igarashi, K. Aikyo, and H. Maeno, Satellite observation of medium scale traveling ionospheric disturbances over Syowa Station, Proc NIPR Symposium Upper Atmosphere, 1, 192-198, 1988.

Otsuka, Y., K. Suzuki, S. Nakagawa, M. Nishioka, K. Shiokawa, and T. Tsugawa, GPS observations of medium-scale traveling ionospheric disturbances over Europe, Ann. Geophys., 31, 163$172,2013$.

Roberts, D.H., J.A. Klobuchar, P.F. Fougere, and D.H. Hendrickson, A large-amplitude traveling ionospheric disturbance produced by the May 18, 1980, explosion of Mount St. Helens, J. Geophys. Res., 87, 6291-6301, 1982a.

Roberts, D.H., A.E.E. Rogers, B.R. Allen, C.L. Bennett, B.F. Bukker, P.E. Greenfield, C.R. Lawrence, and T.A. Clark, Radio interferometric detection of a traveling ionospheric disturbance excited by Explosion of Mount St. Helens, J. Geophys. Res., 87 (A8), 6302-6306, 1982b.

Row, R.V., Acoustic-gravity waves in the upper atmosphere due to a nuclear detonation and an earthquake, J. Geophys. Res., 72 (5), 1599-1610, 1967.

RTO Technical Report TR-IST-051, Characterising the Ionosphere. Final Report of Task Group IST-051, Published January 2009. 1.1.1.2 (Hawlitschka Stefan). 1.3.8 (Prikryl Paul).

Senior, A., M.J. Kosch, T.K. Yeoman, M.T. Rietveld, and I.W. McCrea, Effects of high latitude atmospheric gravity wave disturbances on artificial HF radar backscatter, Ann. Geophys., 24, 2347-2361, 2006

Shashunkina, V.M., Ionospheric effect of the sudden beginning of a magnetic storm on July, 15th, 1959, Geomagn. Aeron. (in Russian), 6 (1), 146-149, 1966.

Shashunkina, V.M., Study of planetary ionospheric effect of the sudden beginning of a magnetic storm, Ionosf. Issled. (in Russian), 16, 91-96, 1968a.

Shashunkina, V.M., Variations of ionospheric parametres during effect SC depending on solar and magnetic activity according to the Lindau-data, Geomagn. Aeron. (in Russian), 8 (2), 352-354, 1968b.

Shashunkina, V.M., Results of study of ionospheric effect of the sudden beginning of a magnetic storm, Ionosf. Issled. (in Russian), 20, 154-165, 1972.

Song, Q., F. Ding, W.X. Wan, L.B. Liu, and B.Q. Ning, Monitoring nighttime medium-scale traveling ionospheric disturbances using the GPS network over North America, J. Geophys., 54, 162-168, 2011.

Taran, V.I., Yu.I. Pod'yachii, V.I. Golovin, V.I. Vashchenko, and I.D. Arkad'ev, Traveling ionospheric disturbances detected using the incoherent scatter method, Ionos. Issled., 27, 102$110,1979$.

Taran, V.I., Yu.I. Pod'yachii, A.N. Smirnov, and L.J. Gerstein, Disturbances of the ionosphere after a ground level burst on supervision by a method of incoherent scatter, Izvest. akadem. nauk SSSR, Phisica Zemly (in Russian), 11, 75-79, 1985.

Tsugawa, T., N. Kotake, Y. Otsuka, and A. Saito, Medium-scale traveling ionospheric disturbances observed by GPS receiver network in Japan, A short review GPS Solutions, 11 (2), 139-144, DOI: 10.1007/s10291-006-0045-5 Key: citeulike:1207528, 2007a.

Tsugawa, T., Y. Otsuka, A.J. Coster, and A. Saito, New characteristics of medium-scale traveling ionospheric disturbances detected with dense wide-coverage TEC maps over North America, Geophys.Res. Lett., 34, 22-101, DOI: 10.1029/2007GL031663, $2007 \mathrm{~b}$.

Tyrnov, O.F., Yu.P. Fedorenko, and L.F. Chernogor, Studying wavelike disturbances of electron density using radio sounding of the ionosphere by coherent signals of navigation satellites, Usp. Sovrem. Radioelektron., 1, 36-80, 2005.

Vadas, S.L., and G. Crowley, Sources of the traveling ionospheric disturbaces observed by the ionospheric TIDDBIT sounder near Wallops Island on 30 October 2007, J. Geophys. Res., 115 (A07324), 1-24, 2010.

Vlasov, A., K. Kauristie, M. van de Kamp, J.-P. Luntama, and A. Pogoreltsev, A study of traveling ionospheric disturbances and atmospheric gravity waves using EISCAT Svalbard Radar IPYdata, Ann. Geophys., 29, 2101-2116, 2011.

Wexler, H., and W.A. Hass, Global atmospheric pressure effects of the October 30, 1961, explosion, J. Geophys. Res., 67 (10), 3875$3879,1962$. 
Yakovets, A.F., V.V. Vodyannikov, G.I. Gordienko, J.F. Ashkaliev, Yu.G. Litvinov, and S.B. Akasov, The response of a night middlelatitude ionosphere to passage of an atmospheric gravitational wave, Geomagn. Aeron., 48 (4), 534-541, 2008.
Yeh, K.C., and C.H. Liu, Acoustic-gravity waves in the upper atmosphere, Rev. Geophys. Space Phys., 12 (2), 193-616, 1974.

Cite this article as: Fedorenko YP, Tyrnov O, Fedorenko V \& Dorohov V: Model of traveling ionospheric disturbances. J. Space Weather Space Clim., 2013, 3, A30. 